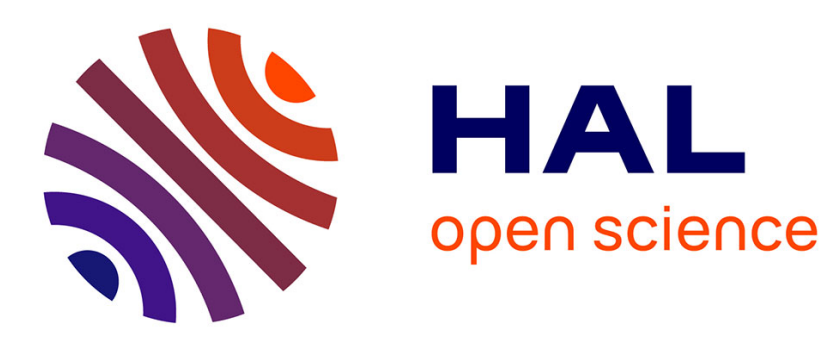

\title{
Advanced numerical prediction of iced airfoil aerodynamics
}

\author{
Michel Costes, Frederic Moens
}

\section{To cite this version:}

Michel Costes, Frederic Moens. Advanced numerical prediction of iced airfoil aerodynamics. Aerospace Science and Technology, 2019, 91, pp.186-207. 10.1016/j.ast.2019.05.010 . hal-02174947

\section{HAL Id: hal-02174947 https://hal.science/hal-02174947}

Submitted on 5 Jul 2019

HAL is a multi-disciplinary open access archive for the deposit and dissemination of scientific research documents, whether they are published or not. The documents may come from teaching and research institutions in France or abroad, or from public or private research centers.
L'archive ouverte pluridisciplinaire HAL, est destinée au dépôt et à la diffusion de documents scientifiques de niveau recherche, publiés ou non, émanant des établissements d'enseignement et de recherche français ou étrangers, des laboratoires publics ou privés. 


\title{
Advanced numerical prediction of iced airfoil aerodynamics
}

\author{
M. Costes ${ }^{1}$, F. Moens ${ }^{2}$ \\ ONERA, The French Aerospace Lab, 92190 Meudon, France
}

The paper presents a numerical investigation of the flow around a NACA23012 airfoil with two ice shapes, a spanwise ridge and leading edge roughness. RANS and URANS simulations are completed with various $1^{\text {st }}$ and $2^{\text {nd }}$ order turbulence models (Spalart-Allmaras, Menter SST, EARSM, DRSM) for a selected number of points of the airfoil polar. To assess the ability of advanced unsteady hybrid RANS/LES models, one selected flow condition of the spanwise ridge case is also computed with a ZDES hybrid method. The results are compared with experimental data (integrated loads, pressure distribution, velocity field) obtained in the ONERA F1 wind tunnel. The Spalart-Allmaras model is the only RANS model among those assessed in this study converging efficiently towards steady-state whatever the flow condition considered. Together with DRSM, it also provides a reasonable predictive effect on the performance degradation due to ice shape. DRSM is much more expensive as it contains more physics, providing natural unsteady solutions which have to be time-averaged. DRSM first and second-order statistic fields compare well with ZDES ones, both of them indicating that geometrical three-dimensionality of the ice shapes should be taken into account. Furthermore, the unsteady content of the DRSM URANS solution is compared with that of ZDES, showing that the vortex shedding phenomenon can also be captured by DRSM.

\footnotetext{
${ }^{1}$ Research Scientist, Aerodynamics Aeroelasticity and Acoustics Department, 8 rue des vertugadins, 92190 Meudon, France.

${ }^{2}$ Research Engineer, Aerodynamics Aeroelasticity and Acoustics Department, 8 rue des vertugadins, 92190 Meudon, France. 


\section{Nomenclature}

\begin{tabular}{|c|c|}
\hline$C_{p}$ & $=$ pressure coefficient \\
\hline$C_{D}$ & $=$ drag coefficient \\
\hline$C_{L}$ & $=$ lift coefficient \\
\hline$C_{\text {Lmax }}$ & $=$ maximum lift coefficient \\
\hline$C_{m}$ & $=$ pitching moment coefficient \\
\hline$c$ & $=$ airfoil chord \\
\hline$d^{+}$ & $=$distance to wall in wall units \\
\hline$f$ & $=$ frequency of pressure fluctuations \\
\hline$G(f)$ & $=$ power spectral density of pressure fluctuation \\
\hline$L / D$ & $=$ lift-to-drag ratio \\
\hline$U_{\infty}$ & $=$ freestream velocity \\
\hline$U_{u}$ & $=$ velocity on top of shear layer \\
\hline$U_{l}$ & $=$ velocity at bottom of shear layer \\
\hline$\zeta$ & $=$ coordinate along normal to shear layer \\
\hline$S t=\frac{f c}{U_{\infty}}$ & $=$ Strouhal number based on airfoil chord \\
\hline$S t_{\delta_{\omega}}=\frac{f \delta_{\omega}}{U_{\infty}}$ & $=$ Strouhal number based on vorticity thickness \\
\hline$\delta_{\omega}=\left(U_{u}-U_{l}\right) /(|\partial U / \partial \zeta|)_{\max }$ & $=$ vorticity thickness \\
\hline$\tilde{v}_{w}^{+}$ & $=$eddy viscosity at wall (roughness model) \\
\hline DDES & $=$ Delayed Detached Eddy Simulation \\
\hline DES & $=$ Detached Eddy Simulation \\
\hline DRSM & $=$ Differential Reynolds Stress Model \\
\hline EARSM & $=$ Explicit Algebraic Reynolds Stress Model \\
\hline ESGH & $=$ Equivalent Sand Grain Height \\
\hline
\end{tabular}




$\begin{array}{ll}\text { HRL, HRLES } & =\text { Hybrid RANS LES } \\ \text { LES } & =\text { Large Eddy Simulation } \\ \text { PSD } & =\text { Power Spectral Density } \\ \text { RANS } & =\text { Reynolds-Averaged Navier-Stokes } \\ \text { RMS } & =\text { Root Mean Square } \\ \text { SA } & =\text { Spalart-Allmaras } \\ \text { SST } & =\text { Shear Stress Transport } \\ \text { URANS } & =\text { Unsteady RANS } \\ \text { ZDES } & =\text { Zonal Detached Eddy Simulation }\end{array}$

\section{Introduction}

Ice accretion induces strong performance degradation of lifting surfaces by modifying the geometry of their leading edge and the state of boundary layers downstream, at the source of premature and highly undesirable flow separation. When aircraft are led to fly in icing conditions it is a serious threat for their safety. Ice protection systems proved their efficiency for maintaining safe flight capabilities in such atmospheric conditions. However the degradation of airfoils and wings performance due to ice-accretion must be considered in case of system failure, runback ice accretion or simply because of ice accumulation during inter-cycle operation of the de-icing system. A wide review and analysis of existing experimental data for iced lifting surfaces was compiled by Lynch and Khodadoust [1], showing the large degradation of performance induced by the various ice-accretion mechanisms. Maximum losses of lift drawn from this extended compilation of results range from $40 \%$ to $80 \%$ of that of clean wing sections, depending on the test condition and configuration. An important aspect emphasized by the authors concerns the uncertainty which results from the various existing experimental databases, most of the time due to Reynolds number effects (which may have a large influence on the clean configuration and therefore on the magnitude of performance degradation) or wind-tunnel wall interferences, so that penalties due to ice-accretion may be underestimated in a significant number of the collected databases. Aircraft designers generally use semi- 
empirical correlations based on such kind of test data to determine performance degradation induced by ice-accretion. Therefore these estimated penalties suffer from the uncertainties mentioned above. Furthermore, as these performance degradations are known to be dependent on the body shape and flow condition, the level of uncertainty is even larger. This is because the effect of ice-accretion on lifting surfaces involves various mechanisms, as discussed by Bragg et al [2], and their effect is highly dependent on the position and geometry of the ice obstacles on the lifting surfaces. In most cases, ice obstacles create recirculation bubbles, and the airfoil performance is highly dependent on its capability to reattach downstream, which is linked to the type of stall of the clean airfoil. They also affect the state of the boundary layer, its evolution further downstream and promote premature trailing edge separation, even when very small geometry modifications are involved due to surface roughness.

The development and validation of accurate methods based on CFD for the prediction of performance degradation due to ice shapes is therefore an important topic of research with great potential benefits for aircraft design and safety. However this is a challenging problem too. Accreted ice blocks are geometrically complex with rough surfaces, and the generation of accurate body conforming grids around such shapes is far from trivial [3]. Furthermore, as ice obstacles are prone to inducing flow separation, an accurate prediction of the flow recirculation zone and of the reattachment line downstream is requested for a proper estimation of the aerodynamic coefficients $C_{L}, C_{D}, C_{m}$ and lift-to-drag ratio $L / D$. As is the case for most separated flows, the Reynolds-Averaged Navier-Stokes (RANS) equations have difficulties to predict such kind of phenomena, due to the lack of universality of the turbulence models used in these situations. On the other hand, the application of more general methodologies such as Large Eddy Simulation (LES) or hybrid RANS-LES (HRLES) to this problem implies considerably more computer resources and therefore only the application of RANS methods for predicting iced airfoil performance is feasible for actual industrial applications. A detailed validation of these various approaches for the simulation of iced airfoil aerodynamic flow is therefore of high interest in order to determine the capabilities and weaknesses of RANS. 
Quite a number of investigators have considered this problem in the past. About 20 years ago, Chung et al [4] performed 2D and 3D RANS simulations with one-equation Spalart-Allmaras (SA) and BaldwinBarth models to investigate a turboprop-powered aircraft accident due to icing. A ridge ice shape located at the leading edge of the wing was considered as it involved the largest performance penalties. Differences between 2D and 3D results were highlighted, but no experiment was available to assess them. Due to the difficulty of the problem, most of the work performed subsequently focused on $2 \mathrm{D}$ configurations, often considering simplified geometries of the ice obstacles. In a series of papers, Dunn et al [5] and Kumar and Loth [6] used adaptive unstructured grids, and Pan and Loth [7] used structured grids, to simulate a quarter-round ice shape to investigate the effect of a spanwise ridge accretion on airfoil performance. Steady-state solutions were considered with the one-equation SA turbulence model. The effect of airfoil geometry, ice obstacle position and height, Mach number and Reynolds number were investigated. A qualitatively good prediction of the available experimental data was found up to stall, but failed to predict the flow when approaching stall and beyond. This was attributed to the unsteady flow developing under these conditions with large-scale vortex shedding which cannot be captured by the model [2]. As a result, higher fidelity simulations were investigated, both in terms of turbulence modeling and of ice geometry. Kumar and Loth [8] as well as Pan and Loth [9] applied the Detached Eddy Simulation (DES) method for similar configurations, showing the better capability of DES to simulate massive separation, in particular the occurrence of a maximum lift coefficient at stall. Nevertheless the agreement with experiment was still limited, which may be explained by the fairly coarse discretizations used. Chi et al [10] compared various turbulence models in a RANS approach to simulate airfoil performance degradation due to realistic ice shapes. The models tested ranged from one-equation SA to seven-equation differential Reynolds stress model (DRSM). For rime ice, where no horn occurs, the SA model was found to be fairly accurate up to stall. Conversely, all models could not predict the large separated regions induced by horns in the glaze ice case, and again the SA model was found to give the best predictions. More recently, Alam et al [11] [12] compared their Hybrid RANS/LES (HRL) model to DES and Delayed Detached Eddy Simulation (DDES) results for an airfoil with horn ice, showing an 
earlier development of flow instabilities than other hybrid models downstream the obstacle. The length of the recirculation bubble was also better predicted, leading to an improved prediction of lift and pressure distribution, although the opposite effect was found for drag. The hybrid methodology also behaved more consistently with grid refinement by comparison to DDES. Duclercq et al [13] applied the Zonal Detached Eddy Simulation method (ZDES) to the computation of the NACA23012 airfoil with a spanwise ridge on both upper and lower surface, showing an improved prediction of the flow separation with respect to RANS. The unsteady nature of the flow with vortex shedding was emphasized. The effect of mesh fineness was also assessed, showing a small influence on results. Similarly, Zhang et al [14] applied the ZDES model to the prediction of iced airfoil aerodynamics, considering both horn and spanwise ridge cases mentioned above, showing the importance of a sufficient spanwise mesh extension in the presence of massively separated flows. Fairly good agreement with experiment was obtained, although none of the computed cases was above the angle of attack at which the $C_{\text {Lmax }}$ occurs. Xiao et al [15] applied DDES with low dissipation scheme to the same horn configuration as Alam and Zhang [11][12][14]. Three grid levels were tested, and the fine grid solution yielded the best pressure plateau prediction in the recirculation by comparison with published work. More recently, the prediction of iced airfoil and wing performance was also considered with the Lattice-Boltzmann methodology ([16] [17]), showing successful predictions for a horn ice shape.

The objective of the present paper is twofold. First a detailed validation of RANS, Unsteady RANS (URANS) and ZDES models for iced airfoil aerodynamics is presented, focusing on the mean flow prediction with an emphasis on performance degradation due to ice. Second, a comparison of the unsteady content captured by a URANS DRSM model and a hybrid ZDES method for massive flow separation induced by ice is performed in order to draw additional information about flow unsteadiness. To the authors' knowledge, it is the first time that the Reynolds stresses computed by DRSM are compared to those captured by HRLES for such complex configuration with massive flow separation. Furthermore, the analysis of flow unsteadiness is expected to give useful information on the validity and capability of URANS for separated flows. 


\section{Case studies}

In the present work, a detailed wind-tunnel aerodynamic database for icing conditions of the NACA23012 airfoil is exploited. It was generated jointly by NASA, the University of Illinois and ONERA [18] [19]. This database includes more particularly full-scale iced airfoil aerodynamic data obtained in the ONERA F1 pressurized wind-tunnel for six selected ice-accretion geometries: a horn, a spanwise ridge, two roughness and two streamwise ice-accretion shapes. These shapes were selected among the most representatives obtained during full-scale ice-accretion tests in the NASA IRT icingtunnel for the same airfoil. The full-scale aerodynamic model used castings of these selected shapes. These castings represent the 3D geometry of the ice obstacles over $381 \mathrm{~mm}$ span. They were repeated to cover the full $3492 \mathrm{~mm}$ span of the airfoil model in the wind tunnel. The tests in the ONERA pressurized F1 wind-tunnel allowed the Reynolds number to vary from $4.5 \cdot 10^{6}$ to $16.10^{6}$ and the Mach number from 0.1 to 0.28 . Angle-of-attack sweeps were performed for the clean airfoil and the six selected iced cases. Data acquisition included loads and moments, pressure distribution and PIV velocity measurements for some cases. Additional data was also obtained at reduced scale and Reynolds number during tests in the University of Illinois low-speed wind-tunnel.

The two ice shapes considered in the present study, a spanwise ridge and a roughness case, are shown in Figure 1. The spanwise ridge (left) typically results from water flowing downstream leading-edge ice protection systems and freezing further downstream. It induces large flow recirculation and consequently severe performance penalties. It should be noted that, although the ice shape actually tested presented geometrical variations in span, a geometrically 2D simulation of the problem was considered, corresponding to the $43 \%$ span section which is equipped with pressure transducers. This particular configuration was also considered by Zhang et al in [14]. It was also used by Duclercq et al [13] to validate their ZDES simulation and the present work is a follow-on of this activity. The roughness shape (right) corresponds to the early stages of ice accretion or it appears during the inter-cycle operation of the ice protection system. Although the original geometry of the NACA 23012 airfoil is weakly altered by 
roughness, the modifications in the leading edge region are larger than the local boundary layer thickness. Consequently, the development of the boundary layer downstream is affected by roughness, leading to premature flow separation with respect to the clean airfoil. Again, a 2D section of the actual roughness was considered in the present work, although the problem is highly $3 \mathrm{D}$ at the scale of the roughness. The present article is a synthesis of a long-term research work whose progress papers were presented in [20], [21] and [22]. The test conditions considered correspond to a freestream Mach number equal to $\mathrm{M}_{\infty}=0.2$ and a Reynolds number of $\operatorname{Re}=15.74 .10^{6}$ based on airfoil chord (equal to $1.829 \mathrm{~m}$ ) and freestream velocity.
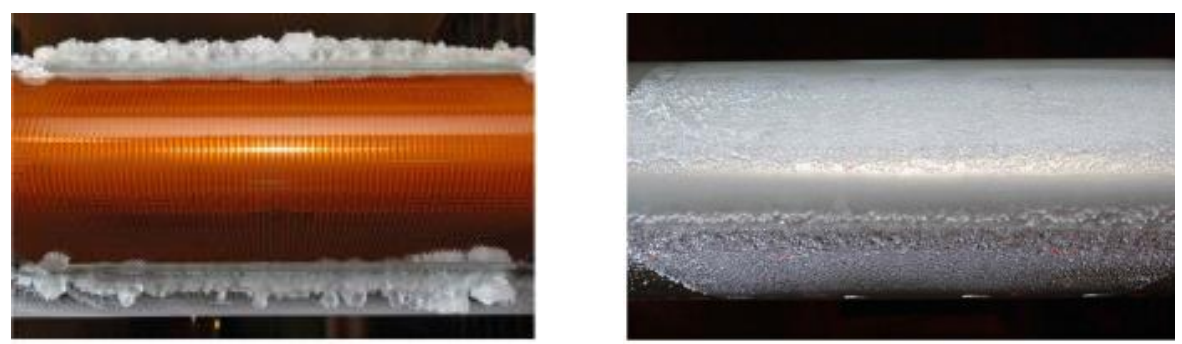

Figure 1: NACA23012 with ridge (EG1159) and roughness (EG1126) ice shape (pictures from accretion tests at IRT)

\section{Numerical method}

The CFD method used in the present work is the elsA multi-purpose software of ONERA for aerodynamics [23]. It solves the compressible flow equations for structured multiblock grids in a finitevolume approach. For space discretization, standard $2^{\text {nd }}$-order centred discretization with Jameson scalar artificial viscosity is used for all RANS and URANS simulations. In the ZDES computation, the upwind AUSM+(P) scheme developed by Edwards and Liou [24] was selected for the inviscid part of the fluxes, mainly because of its low numerical dissipation in the boundary layer. In all cases, the viscous fluxes use a classical centred formulation. To converge the steady RANS equations, a simple backward Euler method with local time-stepping is used. The implicit system is obtained by flux linearization and solved with LU factorization. When possible, multigrid techniques were used to accelerate convergence to steady-state. For time-accurate URANS computations, second-order implicit time-discretization is applied using the dual time-stepping method, with LU factorization too. The number of dual-time iterations 
depends on the problem considered, and is generally selected in order to ensure about one order of magnitude reduction of the dual-time residuals. Finally, the ZDES method uses Gear implicit timediscretization scheme which is again solved iteratively by flux linearization, LU factorization and Newton subiterations.

A wide range of turbulence models are available in elsA, from algebraic models to LES. In the present work, models of increasing complexity have been considered for validation: the one-equation SA model [25], Menter two-equation k- $\omega$ model with SST correction [26], Hellsten two-equation EARSM k- $\omega$ model [27], and the SSG- $\omega$ or SSG/LRR- $\omega$ seven-equation DRSM model [28] [29]. The first two models follow Boussinesq's hypothesis of linear dependence of the Reynolds stresses to the velocity gradient tensor. The third one belongs to the class of nonlinear eddy viscosity models, still considering two transport equations only. The last one is computationally more expensive as it describes the transport of the six Reynolds stresses and of one additional variable related to the turbulent length scale, in the present case, the widely used specific dissipation rate $\omega$. Most computations were started from a constant freestream state. As the SSG- $\omega$ DRSM model does not generally develop its own turbulence starting from freestream state, it was initialized from a SST simulation, contrary to the SSG/LRR- $\omega$ which was started from a uniform freestream state.

Additionally, ZDES simulations [30] of the spanwise ridge case were also considered at a single incidence angle. This technique was originally developed by Deck to get a flexible and efficient framework to cope with practical issues encountered with technical applications [31]. It also aims at reducing the grid-induced separation phenomena which can be obtained with the original DES method [32]. The ZDES method is zonal, allowing one to select the areas of the computational domain where either RANS or DES are applied according to several formulations available. Schematically, three modes have been defined, corresponding to three physics of flow separation: mode 1 corresponds to a separation induced by geometry, mode 2 implements the separation due to the pressure gradient over a smooth surface, and mode 3 also accounts for the state of the upstream boundary layer. These modes correspond to different definitions of the length scales which discriminate the RANS and LES domains of the DES 
zones. In the present computation, ZDES mode 2 (i.e. the "automatic" mode of ZDES) was selected in order to compute flow reattachment properly. Finally, the subgrid length scale used in the present computations is also based on vorticity to facilitate the generation of turbulence along the shear layer right downstream the top of the spanwise ridge.

\section{Description of grids}

\section{EG1159 case}

The grids used for the RANS and URANS simulations are based on a 2D version of a mesh previously used by Duclercq et al [13] to perform a ZDES simulation of the same configuration. This multiblock structured grid was generated with ICEM-CFD Hexa (Figure 2). The total number of points for the $2 \mathrm{D}$ mesh is equal to 97,873 . It is refined in the vicinity of the ice obstacle in order to resolve flow separation properly. Downstream the ridge, the cells size is based on vorticity thickness $\delta_{\omega}=$ $\left(U_{u}-U_{l}\right) /(|\partial U / \partial \zeta|)_{\max }$ of the shear layer developing at the boundary between the recirculation bubble and the outer flow. The corresponding constraints are $\Delta x=\delta_{\omega} / 2$ chordwise and $\Delta z=\delta_{\omega} / 20$, normalwise. An additional mesh was generated around the upper surface ridge ice shape and the recirculation downstream of it for ZDES, in order to better simulate the separated bubble downstream the spanwise ridge. This second mesh extends towards the airfoil mid-chord and includes 108,192 points

(Figure 2). It was embedded into the previous airfoil mesh using the Chimera overset grids method, and was used with the SA model to check that the RANS solutions dependence on mesh density is limited (Figure 3). In this figure, results obtained with a coarse multiblock mesh of 42,140 points are also plotted in order to have a wider idea of the effect of mesh refinement, which is actually small. 
a) 2D multiblock grid

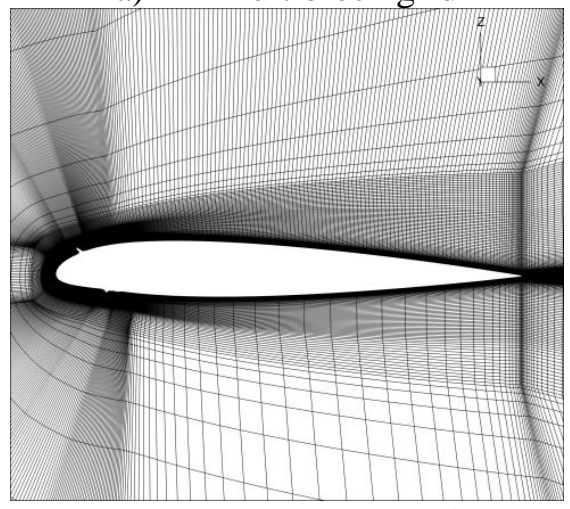

c) ZDES mesh I

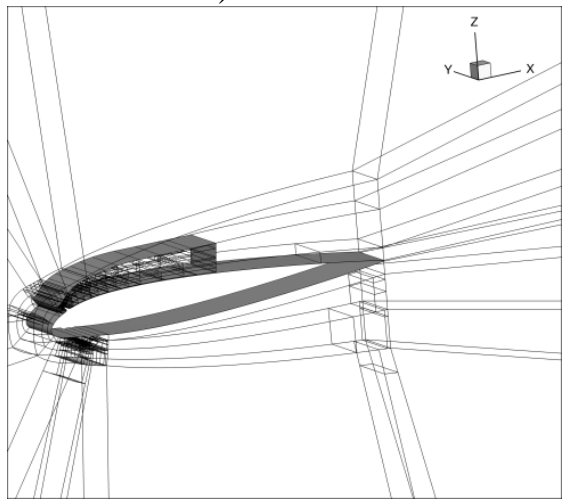

b) Chimera grid

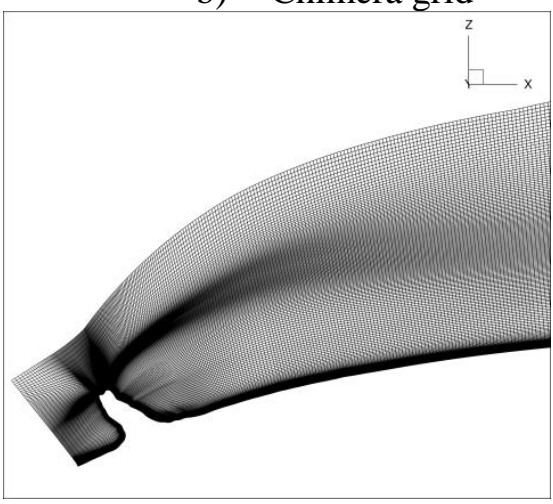

d) ZDES mesh II

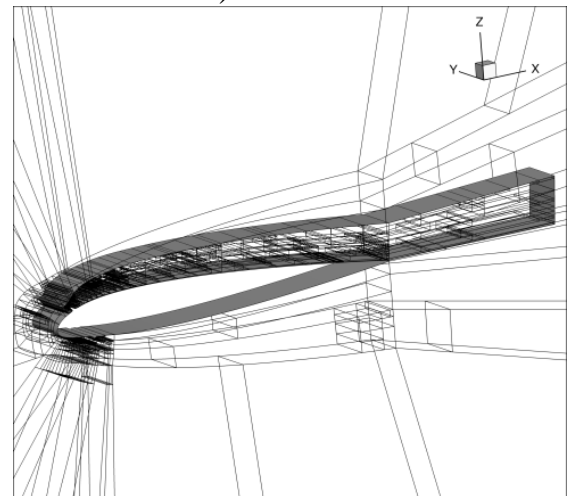

Figure 2: View of meshes used for EG1159 computation
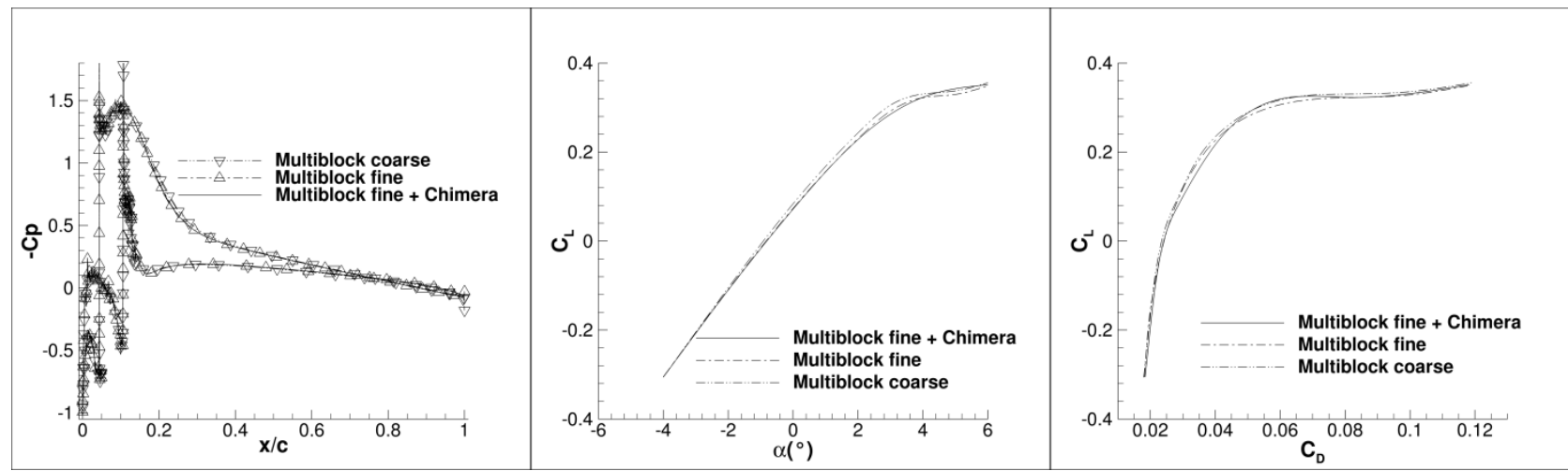

Figure 3: Grid sensitivity of EG1159 solution with SA model - pressure distribution at $\alpha=2^{\circ}$ and airfoil polar curves

For ZDES computations, both 2D meshes were extruded in the spanwise direction, and the Chimera child mesh was progressively refined to comply with DES requirements. The final mesh was extruded over $12.75 \%$ chord in 401 planes for the child mesh on which DES is applied, while the background airfoil mesh was extruded in 50 planes along the same span, with a DES resolution for the upper surface of the airfoil only. This first ZDES mesh, once refinement is achieved, includes 43,027,589 
points. The effect of mesh refinement on the time-averaged solution is significant, as shown by Figure 4. The eddy viscosity computed at mid-span is plotted in the first row, showing a reduction of its maximum value in the DES region as the grid is refined. The bottom row plots the corresponding time-averaged chordwise pressure distribution compared to F1 experiment (symbols). The coarser grid solutions (Figure $4 \mathrm{a}$ and b) show a good correlation with the experimental pressure in the recirculation bubble due to error compensation, and flow separation near the trailing edge. As the grid is refined (Figure $4 \mathrm{c}$ and $\mathrm{d}$ ), the trailing edge separation disappears, the mean pressure in the bubble increases and the recompression after reattachment expands over a longer region than in experiment. This last point is due to intense vortex shedding downstream the bubble. As the DES part of this first mesh stops over the upper surface of the airfoil, an uncertainty remained on the capability of the present simulation to compute flow reattachment properly. A second ZDES mesh was generated by extending the first child mesh half a chord downstream the trailing edge (Figure 2 d). This new ZDES mesh has 59,530,973 points. An instantaneous view of the turbulent viscosity contours in a middle-span cut of the mesh and of the iso-surface of Q criterion is presented in Figure 5 for both child grids. ZDES results capture the fast development of instabilities downstream the spanwise ridge with good spatial resolution. In ZDES mesh I, the end of the child mesh at mid-chord is also clearly noticeable, leading to a saturation of the eddy viscosity contours downstream with larger scale vortices being shed downstream. 
a) $21.0 \mathrm{M}$ cells

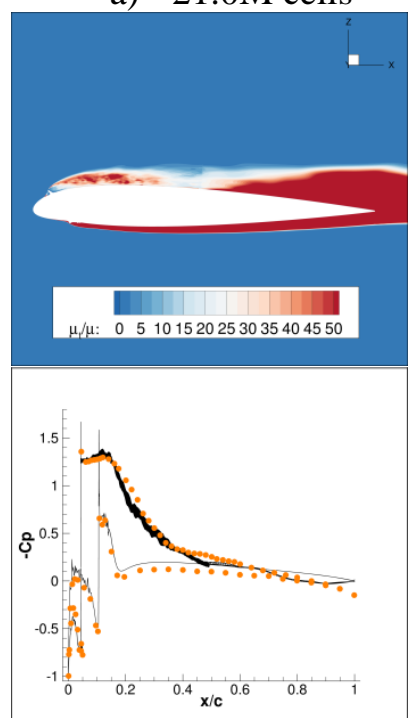

b) $25.3 \mathrm{M}$ cells
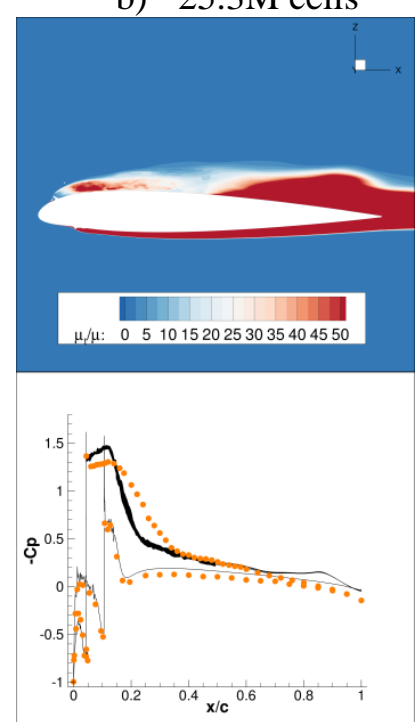

c) $35.5 \mathrm{M}$ cells
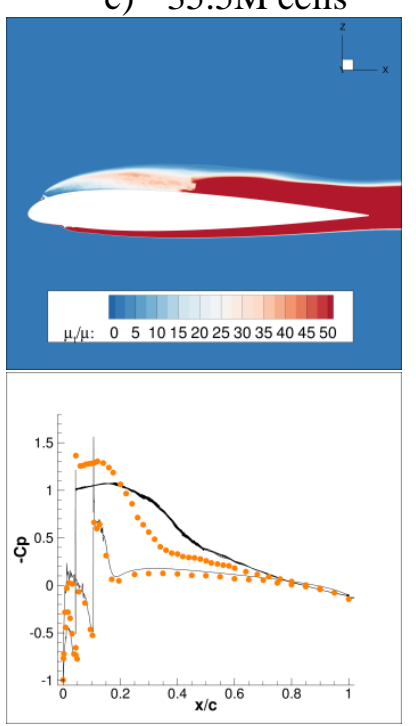

d) $39.7 \mathrm{M}$ cells
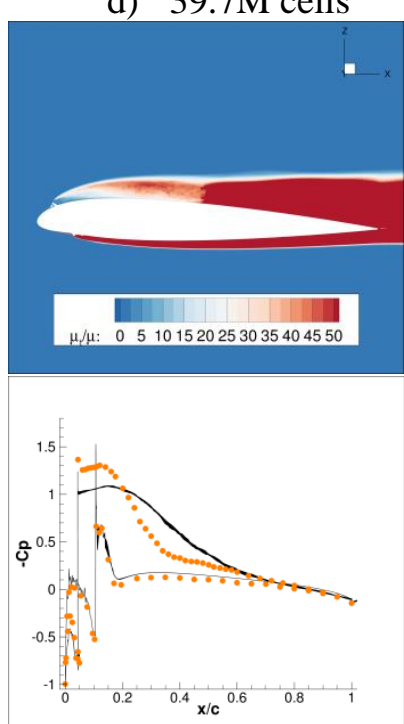

Figure 4: Effect of grid refinement on time-averaged subgrid viscosity and chordwise pressure distribution (ZDES mesh I)
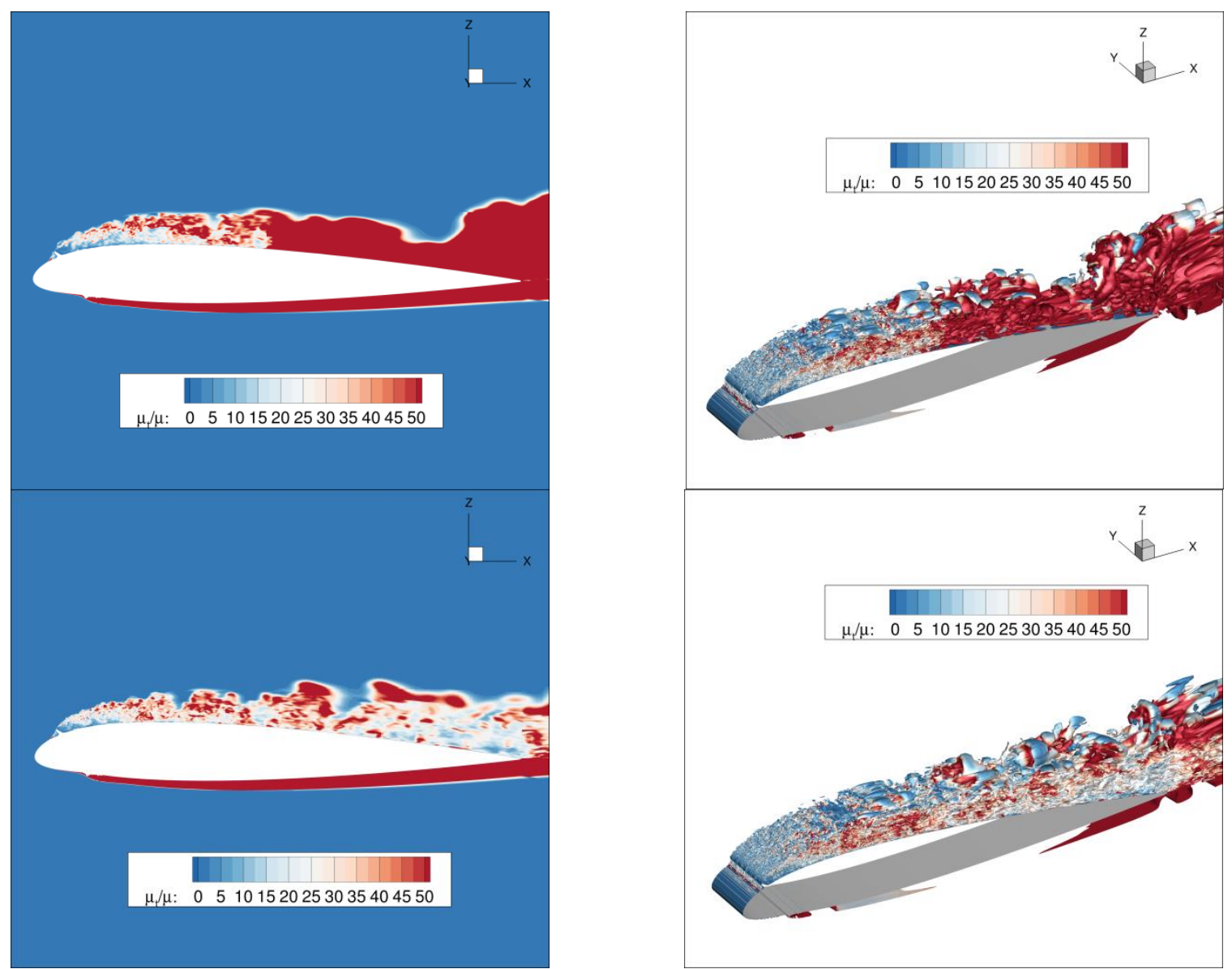

Figure 5: Contours of instantaneous eddy viscosity and iso surface of Q criterion colored by turbulent viscosity in mid-plane for ZDES mesh I (top) and II (bottom) 


\section{EG1126 case}

Two sets of grids were generated to simulate this case, one set corresponding to the airfoil with roughness assumed to be purely two-dimensional, the other one for the clean airfoil (Figure 6). As for the previous case, they were generated with ICEM-CFD Hexa. For the airfoil with roughness, three successive grids were generated with increasing refinement in the boundary layer. At $\alpha=10^{\circ}$ incidence, the number of points in the upper surface boundary layer is in the range 60 to 80 for the first mesh, 80 to 100 for the second mesh and 120 to 140 for the third mesh. On the lower surface, the corresponding number of points equals 40 to 60 for the first mesh, 60 to 80 for the second mesh and 80 to 100 for the third mesh. No chordwise refinement was done, although it would have allowed a better description of roughness details. The effect of this parameter was investigated by Chung et al in [33]. They concluded that from $50 \%$ of the roughness geometry details included the effect on aerodynamic results is small. Thus it can be estimated that the present chordwise discretization of leading edge roughness is sufficient for our purpose. As shown in Figure 7, the sensitivity of solutions to grid was found to be very small and the finer mesh was finally retained, including 151,602 points. The same discretization was kept for the clean airfoil which was used for computing clean airfoil aerodynamic characteristics and also to apply the roughness model in the SA turbulence model.
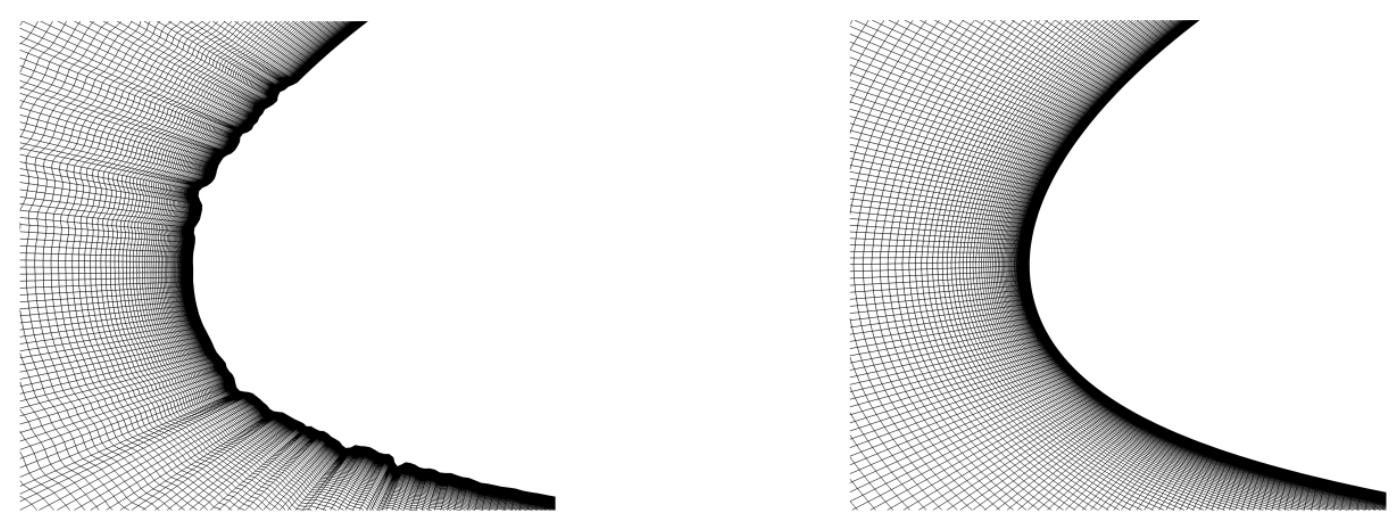

Figure 6: Leading edge detail of meshes used for EG1126 computation - gridded roughness (left), clean airfoil (right) 

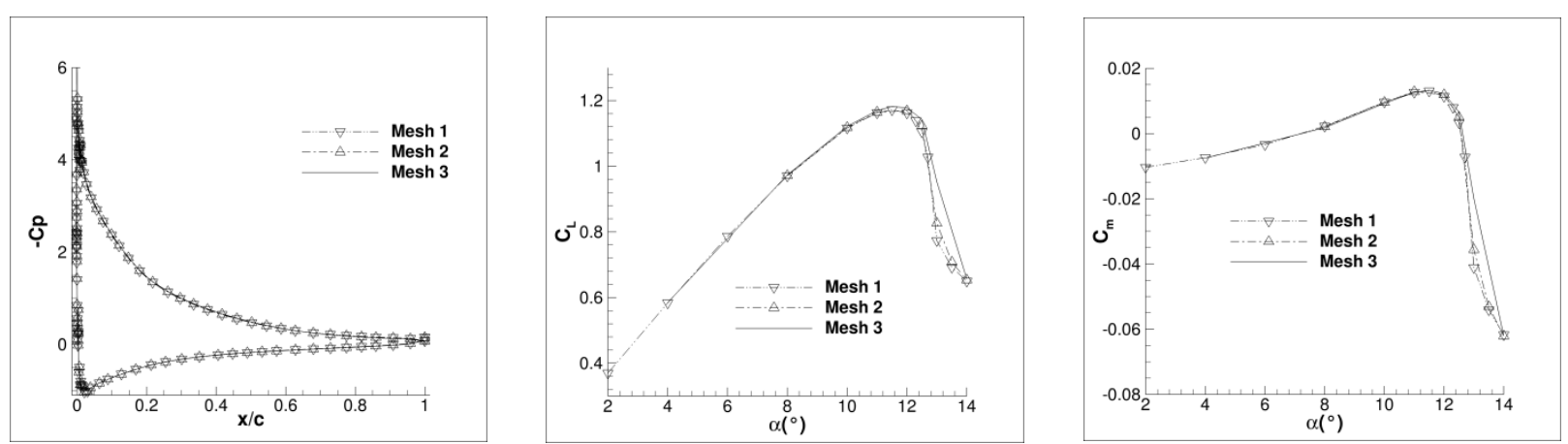

Figure 7: Grid sensitivity of EG1126 solution with SA model - pressure distribution at $\alpha=12^{\circ}$

\section{Time averaged results}

\section{Effect of turbulence modelling for EG1159}

\section{Flow Convergence}

In the available test data, incidence angles between $-4^{\circ}$ to $+6^{\circ}$ were considered for the numerical simulations, focusing mainly on the case $\alpha=2^{\circ}$ for which the available experimental data also includes PIV. The various turbulence models tested (Spalart-Allmaras, Menter k- $\omega$ SST, Hellsten's EARSM k- $\omega$, DRSM SSG- $\omega$ ) and the ZDES simulations over the two Chimera grids were thus all run for a common angle of attack $\alpha=2^{\circ}$. While the ZDES method is naturally unsteady and requires a time-consistent resolution method, all RANS models are expected to provide steady solutions, although the occurrence of flow separation may render the convergence difficult. The SA model is the only one converging towards steady-state in a local time-stepping approach, providing more than 7 orders of magnitude residual reduction. Conversely, the other RANS models do not converge properly. The SST model shows a reduction of residuals of less than 4 orders of magnitude and the resulting integrated loads show large amplitude oscillations. Concerning the EARSM model, less than 3 orders of magnitude residual reductions are obtained together with a large amplitude oscillation of integrated loads similarly to SST. The SST and the EARSM model do converge to steady-state when a time-consistent global time-stepping approach is used, which is very CPU time-consuming. Both models converge towards constant values of the lift and drag coefficient, provided that they are run for sufficiently long time, typically 50 to 60 convective times. Finally, the DRSM model provides quite a modest residual reduction as it is initialized 
from a SST flow solution, and the corresponding result is not converged yet, showing large amplitude oscillations. This model also requires a time-accurate computation, but the solution obtained is naturally unsteady (see Figure 8 a for the evolution of lift coefficient). The unsteady solution shows large amplitude oscillation of the lift. It will be discussed more in details below. For all these unsteady computations, the choice of the physical time step was driven by the capability of the dual-time iterations to converge properly. They typically varied between $4.10^{-8} \mathrm{~s}$ for the DRSM simulation and $2.10^{-6} \mathrm{~s}$ for the EARSM and the SST computation.

In the case of ZDES, the physical time step was set to $4.10^{-7}$ s, providing about 2 orders of magnitude in residual convergence in five Newton iterations. The corresponding ZDES results capture the unsteadiness of the turbulent flow developping downstream the ridge ice shape. Instabilities develop very rapidly downstream the ice obstacle as shown by the contours of Q criterion in Figure 5. As a result, the time evolution of the lift coefficient presents a combination of low and high frequency fluctuations for both overset grids used, with a global evolution of this parameter seeming more erratic for mesh II (Figure $8 \mathrm{~b}$ and $\mathrm{c}$ ). This is probably related to the larger downstream extension of the ZDES mesh II, as the transition between the child mesh I and the background mesh seems to promote larger scale vortex shedding along the downstream half chord of the airfoil. However, large vortical structures develop downstream the reattachment of the shear layer in both cases.

a) DRSM

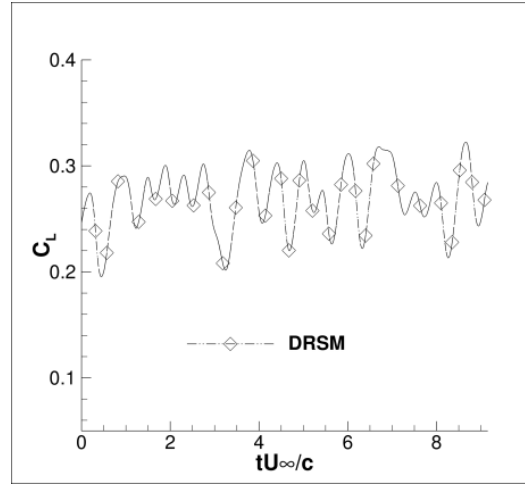

b) ZDES Mesh I

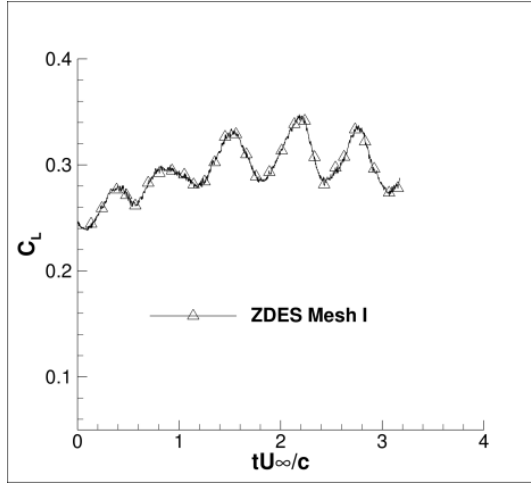

c) ZDES Mesh II

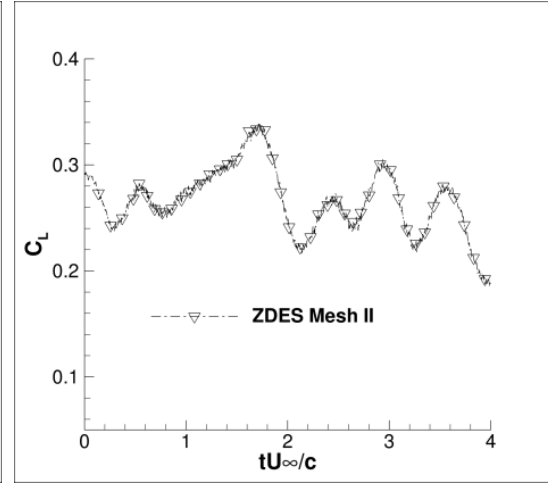

Figure 8: Evolution of lift coefficient versus convective time for ZDES and DRSM computations at $\alpha=2^{\circ}$ 


\section{Comparison of mean solutions at $\alpha=2^{\circ}$}

For comparing the various results, the ZDES and DRSM outputs were time-averaged over a period of time as long as possible to obtain a meaningful mean aerodynamic field. They correspond approximately to nine convective times for the DRSM simulation and three to four convective times for the ZDES simulations. Additionally, the ZDES computations were space-averaged along the span direction. It is clear from Figure 8 that a longer period of time would be necessary to get good statistical convergence of the ZDES simulations on both grids. However, the examination of mean results in the course of the computations tend to show that these time-averaged results are meaningful, as also discussed below in the paragraph devoted to statistical convergence.
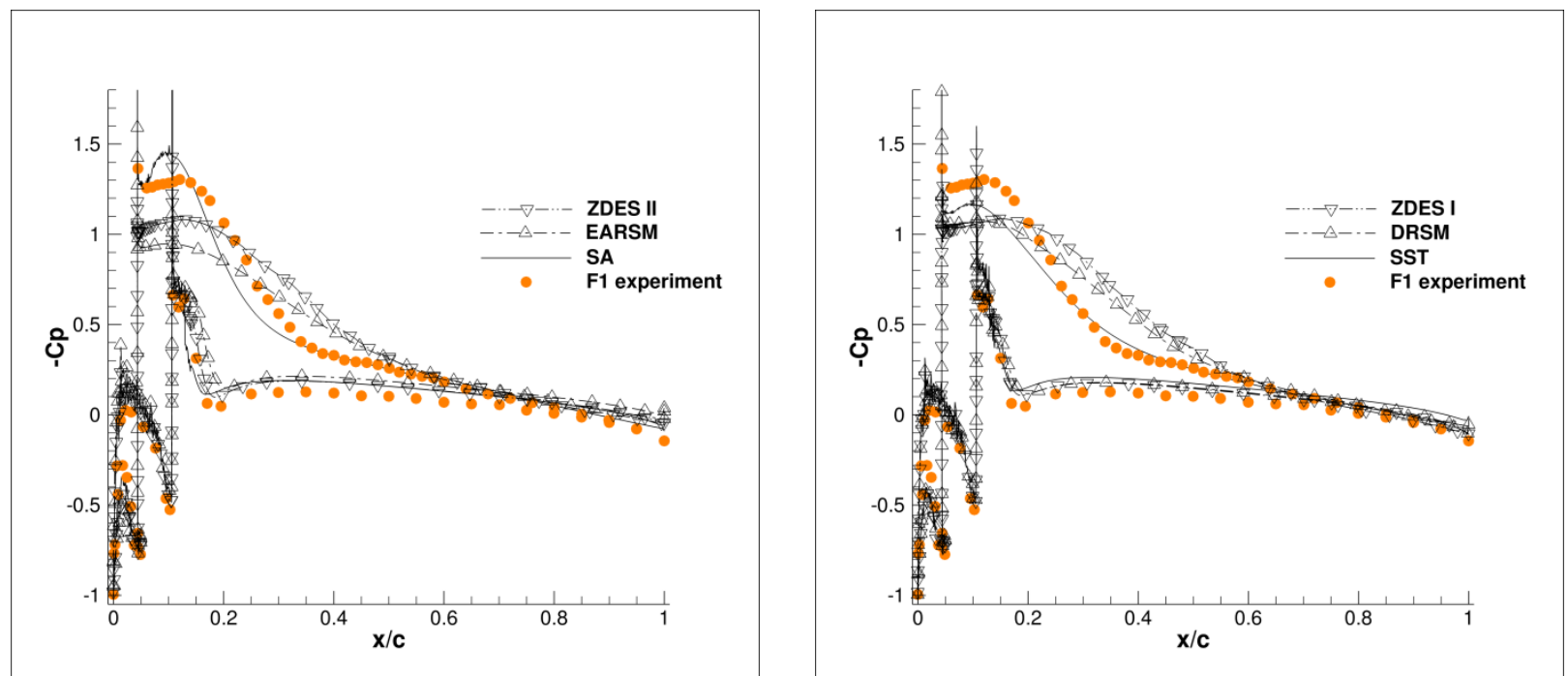

Figure 9: Comparison of pressure distribution for various turbulence models (EG 1159, $\alpha=2^{\circ}$ )

The pressure distributions are compared with F1 experiment in Figure 9. The SA and SST models predict the higher suction on the upper surface of the airfoil, in closer agreement with experiment. However, the pressure plateau in the recirculation is not correctly predicted by the SA model with a too high suction in the middle of the recirculation and an earlier recompression downstream. On the other hand, the SST model overestimates the pressure level in the recirculation zone, with a small acceleration inside the recirculation and a too smooth recompression downstream with flow close to separation in the vicinity of the trailing edge. This has an impact on the whole pressure distribution on the lower surface which provides less satisfactory results than the SA ones. The EARSM model still deteriorates the situation as 
larger flow separation is obtained over the airfoil upper surface. As a result, the suction induced by the spanwise ridge is the lowest of all together with the smoothest recompression downstream, providing a poor correlation with experiment. The DRSM and both ZDES results share many points in common. The lower surface pressure is correctly predicted in all cases, giving a correlation with experiment as good as the SA one. On the contrary, the upper surface pressure distribution does not fit the experimental results. The pressure level in the recirculation bubble is too high, a little above the one predicted by SST, but the length of the bubble (about 10\% chord) with a relatively constant pressure seems correct. However, the pressure rise in the reattachment zone downstream is too slow, the computed pressure approximately reaching the experimental one between $50 \%$ and $55 \%$ chord only. This is certainly explained by the intense vortex shedding predicted by these simulations downstream reattachment, as shown in Figure 10 where samples of instantaneous pressure data are plotted. For all these samples, the beginning of pressure rise is correct and pressure oscillations occur slightly downstream. Time averaging smoothes out these pressure oscillations, but at the same time they reduce the pressure gradient by spreading the recompression. This phenomenon is certainly also at hand in the experiment, but it is much less noticeable as the pressure rise is sharper. This tends to show that the vortex shedding is too intense in these unsteady simulations. This may come from three-dimensional effects not considered in the simulation, since the ice geometry shown in Figure 1 is clearly three-dimensional. Another possibility for the ZDES simulations could be that the spanwise extension of the mesh is too small for the present simulation. However, Duclercq et al did not find any significant effect of spanwise extension from $8.5 \%$ chord to $34 \%$ chord for this same case [13]. 
a) DRSM

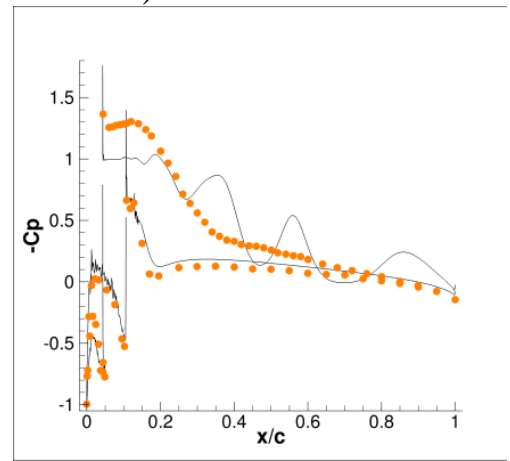

b) ZDES mesh I

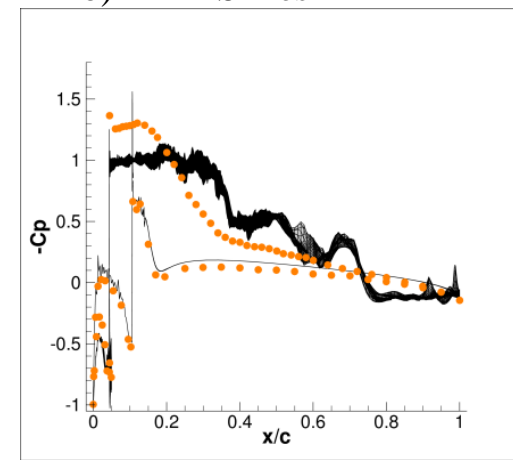

c) ZDES mesh II

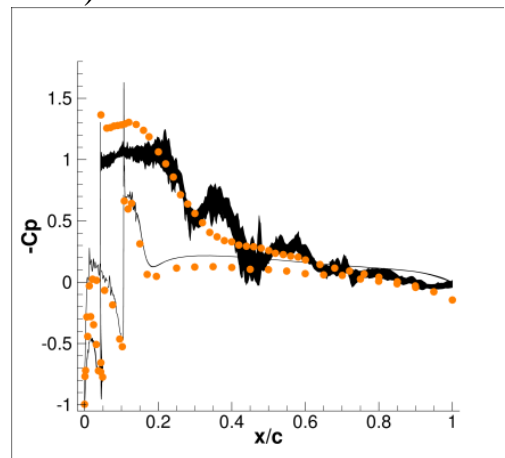

Figure 10: Examples of instantaneous pressure distribution for DRSM and ZDES simulations

A comparison of the velocity contours with PIV comfort these observations (see Figure 11 where only selected results are plotted). The SA model tends to predict a too short recirculation, but the velocity field downstream fits fairly well the PIV results. DRSM and ZDES yield very similar mean velocity contours, with a recirculation bubble larger than the one predicted with SA, and more importantly a larger velocity defect than in the PIV data downstream. Though not shown here, the SST and EARSM results predict an even larger velocity defect, consistently with the pressure distributions. 
a) $\mathrm{SA}$

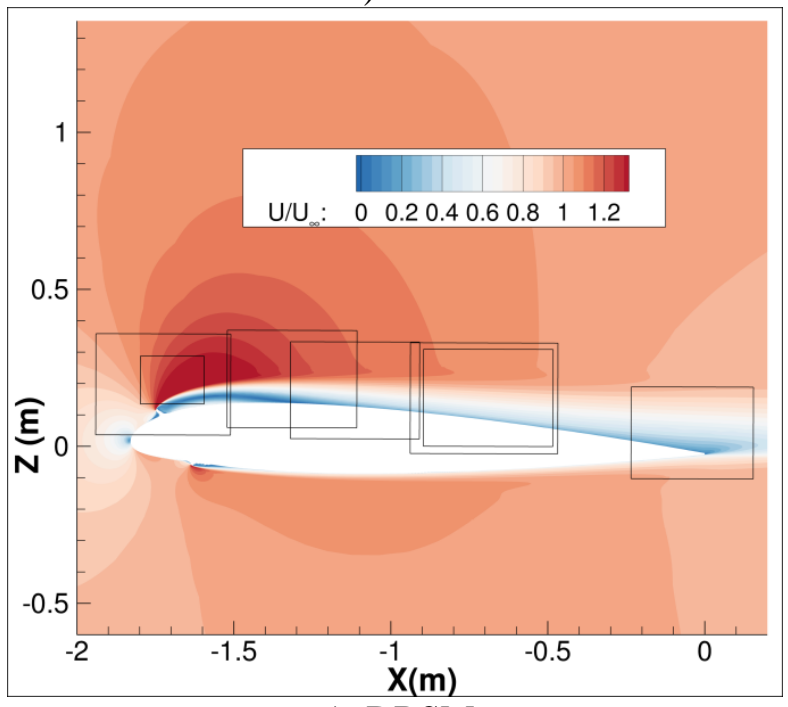

c) DRSM

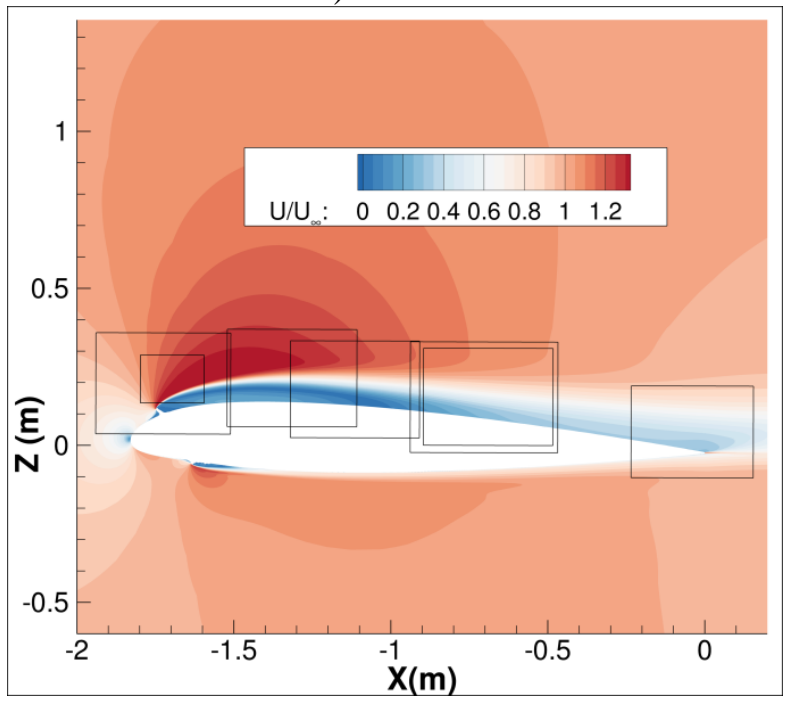

b) PIV

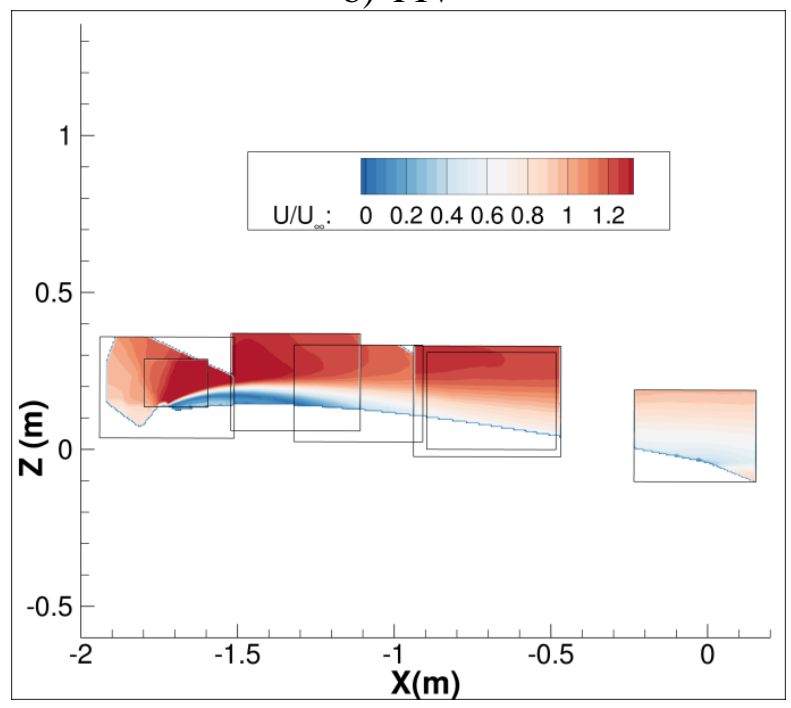

d) ZDES mesh II

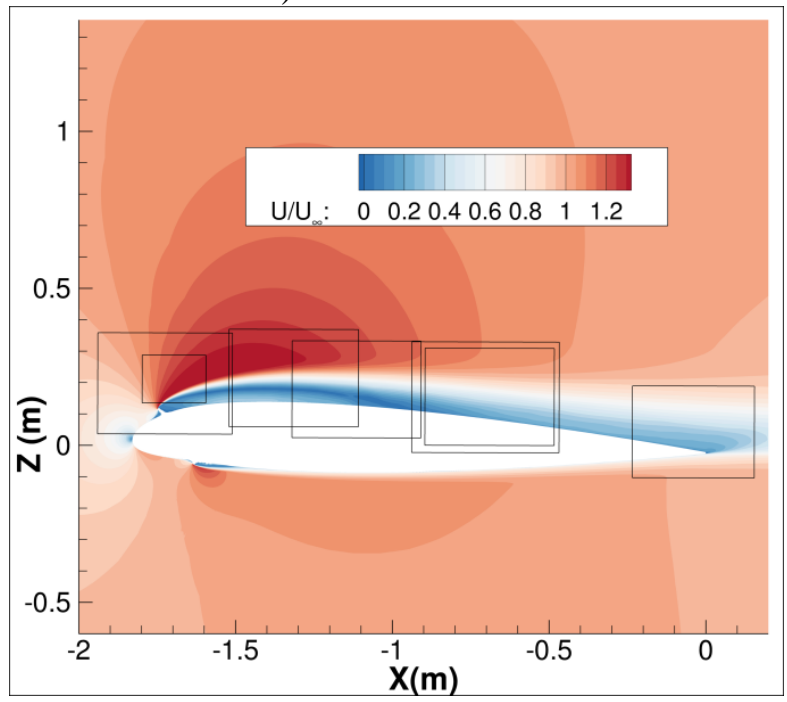

Figure 11: Comparison of mean velocity magnitude for selected models (EG 1159, $\alpha=2^{\circ}$ )

\section{Comparison of pressure distributions for other incidences}

The pressure distributions obtained at $\alpha=0^{\circ}$ and $\alpha=4^{\circ}$ with SA, SST and DRSM are presented in Figure 12. Basically the same qualitative behavior is obtained as at $\alpha=2^{\circ}$. For both cases, the SA model allows a very good convergence to steady-state, the SST model requires global time-stepping to yield a converged steady solution and the DRSM model provides unsteady solutions with vortex shedding downstream the ridge ice shape, so that the flow must be time-averaged for comparison with the other solutions and experiment. 
At $0^{\circ}$ incidence the correlation with experiment is generally quite good, but the computed pressure distributions show similar trends as at $2^{\circ}$ incidence. All models predict reasonably well the pressure distribution on the lower surface, and they mainly differ on the upper surface downstream the spanwise ridge until reattachment. The pressure level predicted by the SA model in the recirculation bubble is too small with increasing suction until reattachment. For SST, the pressure level inside the bubble is slightly too high but its chordwise evolution is much better predicted than by SA. The recompression downstream the bubble is better predicted too. Finally, the DRSM model predicts too high pressure in the recirculation with again a too smooth pressure rise downstream the bubble. This is again due to a too intense vortex shedding downstream the bubble which does not correspond to the experimental results.

At $\alpha=4^{\circ}$, flow separation is overestimated for all turbulence models, resulting in a poor correlation with experiment. The SA model gives the higher suction right downstream the spanwise ridge, close to the experimental level, but the pressure rise occurs much too early and smoothly downstream where massive flow separation is obtained. SST prediction is qualitatively similar to SA one, with higher pressure in the recirculation. Finally, DRSM still predicts higher pressure in the recirculation followed by higher loading in the separated part of the airfoil due to intense vortex shedding. Such a pressure distribution is closer to what is actually obtained in the experiment at $5^{\circ}$ incidence, showing that airfoil stall is overestimated by the model. 
a) $\alpha=0^{\circ}$

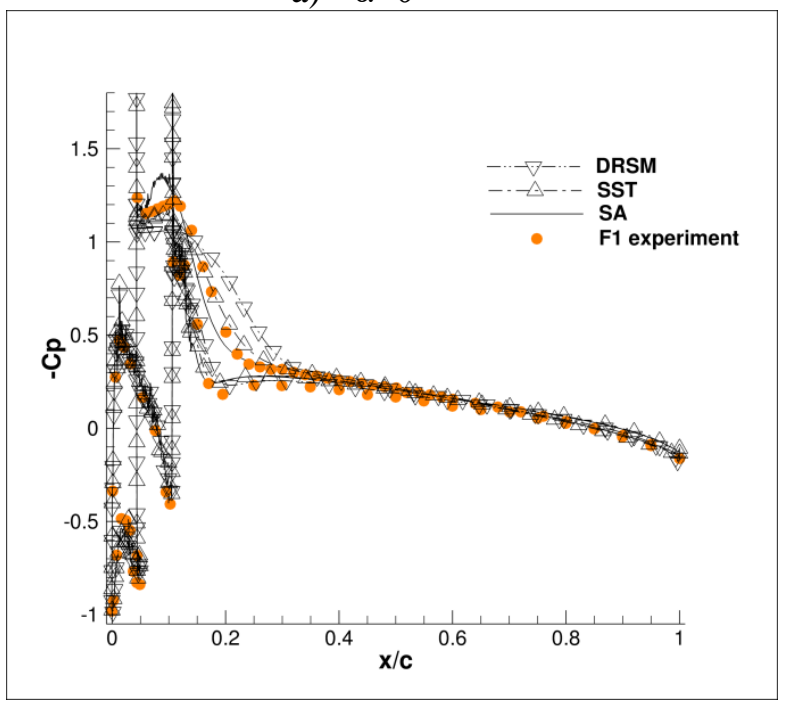

Figure 12: Comparison of pressure distribution for various models b) $\alpha=4^{\circ}$

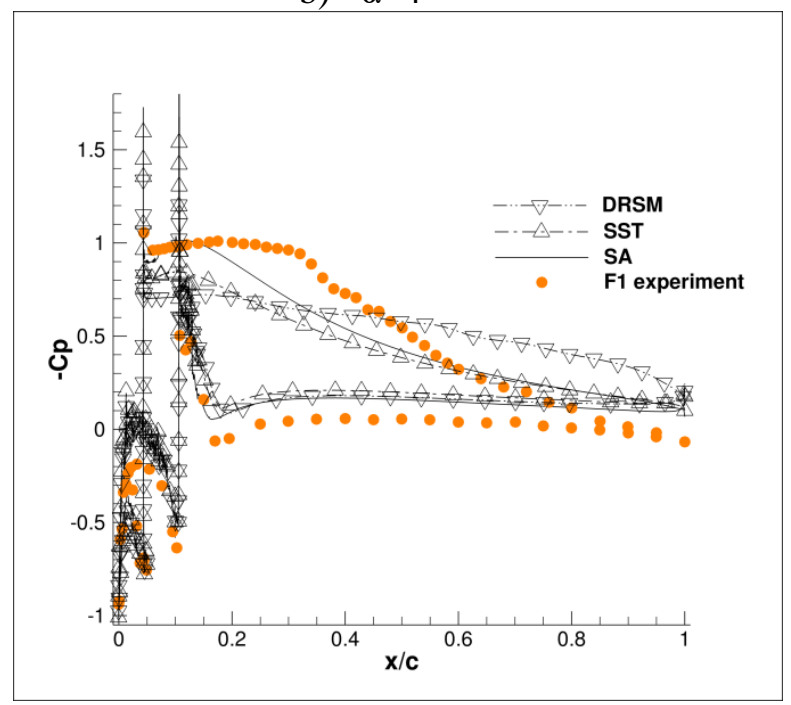

Integrated results

The polar curves of the NACA 23012 airfoil with spanwise ridge computed with the various turbulence modelling techniques are compared to the F1 experiment in Figure 13. The SA model provides a fair description of the airfoil loads. The stall angle is approximately well predicted, although the $C_{\operatorname{Lmax}}$ is underestimated by $30 \%$ (about 0.3 instead of 0.45 ). The drag rise and pitching moment loss after stall are also correctly predicted. On the contrary, the SST model predicts too early stall with a large underestimation of the maximum lift. The only incidence angle computed with the EARSM model is clearly off the polar curves as the airfoil is already stalled at $2^{\circ}$ incidence. The DRSM model is in slightly better agreement with experiment than SA in the linear part of the lift polar, but it tends to stall too early. Finally, the two ZDES results at $2^{\circ}$ incidence are also in fairly good agreement with experiment, similarly to the DRSM results. Other incidences should be considered in order to better assess the capabilities of this methodology to predict iced airfoil performance. However, as discussed before, there is an uncertainty about the actual two-dimensionality of the test data, which makes this validation work more difficult. 

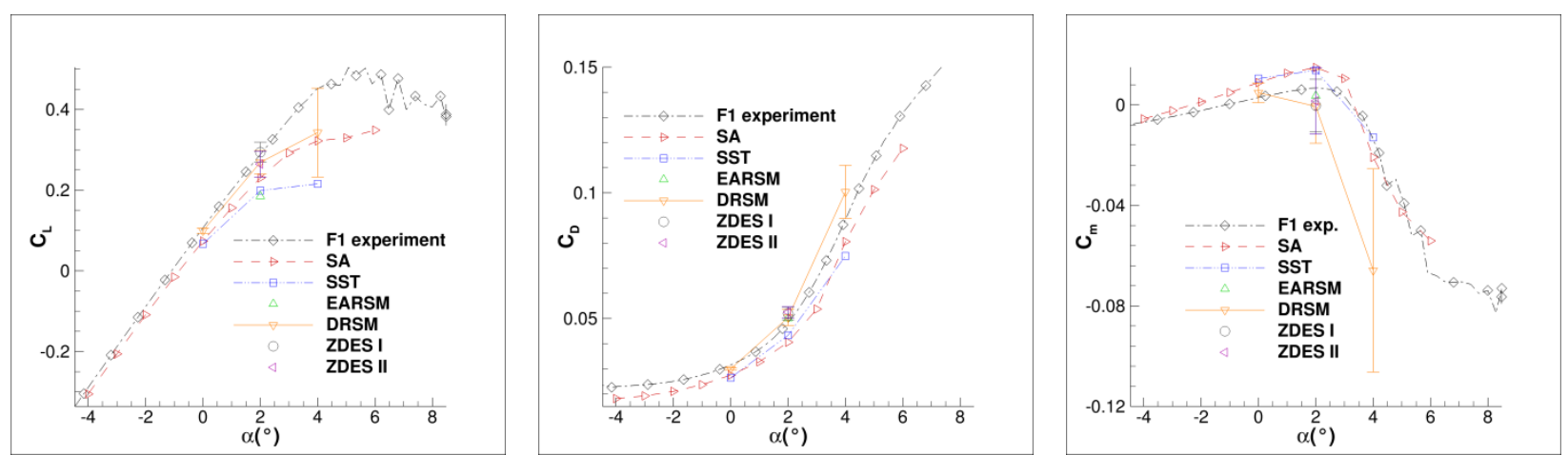

Figure 13: Effect of turbulence model on integrated results for EG1159

\section{Effect of turbulence modelling for EG1126}

\section{Roughness modelling}

For the roughness case, only two turbulence models were tested, namely the SA and DRSM SSG/LRR- $\omega$ models. This DRSM turbulence model was preferred to SSG- $\omega$ due to its better robustness. Its use was motivated by the convergence difficulties encountered in the previous EG1159 simulations. For both models, the roughness shape was included in the grid, assuming a two-dimensional roughness geometry. Additionally, the SA model was applied with a roughness model developed by Aupoix [34]. The model assumes that the roughness height is small with respect to the boundary layer thickness. The eddy viscosity is prescribed to be non-zero at the wall due to roughness. Its value $\tilde{v}_{w}^{+}$, expressed in wall units, explicitly depends on the Equivalent Sand Grain Height (ESGH) $k_{s}^{+}$. The velocity profile is modified in order to follow that of the smooth conditions shifted by a distance $\frac{\widetilde{v}_{w}^{+}}{\kappa}$, where $\kappa$ is the von Karman constant, giving:

$$
d^{+}=d_{\min }^{+}+\frac{\tilde{v}_{w}^{+}}{\kappa}, \tilde{v}_{w}^{+}\left(k_{s}^{+}\right) \Longrightarrow\left[\tilde{v}^{+}\left(y^{+}\right)\right]_{\text {rough }}=\left[\tilde{v}^{+}\left(y^{+}+\frac{\tilde{v}_{w}^{+}}{\kappa}\right)\right]_{\text {smooth }}
$$

The simulations are run on the clean airfoil mesh and the model is applied at the location of the ice roughness (Figure 14). The ESGH was determined by adjustment with gridded roughness simulations at $\alpha=10^{\circ}$. Initial values were obtained from experimental data gathered in the low-speed wind-tunnel of the University of Illinois at Urbana Champaign, where various grit distributions over the clean airfoil have been tested [35], giving $\mathrm{k} / \mathrm{c}=0.0009$ on the lower surface and $\mathrm{k} / \mathrm{c}=0.0013$ on the upper surface. These 
values are consistent with measured ice roughness sizes in [36]. However, such ESGHs do not provide sufficient airfoil performance degradation over the full range of angle of attack with respect to gridded roughness. A multiplying factor was then applied to these initial values in order to obtain the same aerodynamic coefficients as the gridded roughness at $\alpha=10^{\circ}$. It was found that a factor of 4 is required to approximately simulate the $C_{L}, C_{D}$ and $C_{m}$ results of the gridded roughness at this angle of attack, thus giving $\mathrm{k} / \mathrm{c}=0.0036$ on the lower surface and $\mathrm{k} / \mathrm{c}=0.0052$ on the upper surface (Figure 14). The corrected height results are compared to those of the gridded roughness in Figure 15. The corrected surface pressure and boundary layer momentum thickness match those of the gridded roughness quite well, contrary to what was obtained with the initial ESGH definition. However, the agreement is not so good for the skinfriction coefficient, especially in the vicinity of the leading edge, although it is greatly improved compared to uncorrected results. The prescribed ESGH is one order of magnitude larger than the local boundary layer thickness (instead of a factor of 3 to 5 in [36]), so that the model is applied outside of its domain of validity and may thus explain this discrepancy on the skin-friction coefficient. Finally, the corrected roughness height also allows an almost perfect match of the lift polar curve with that of the gridded roughness up to stall, as will be seen later.
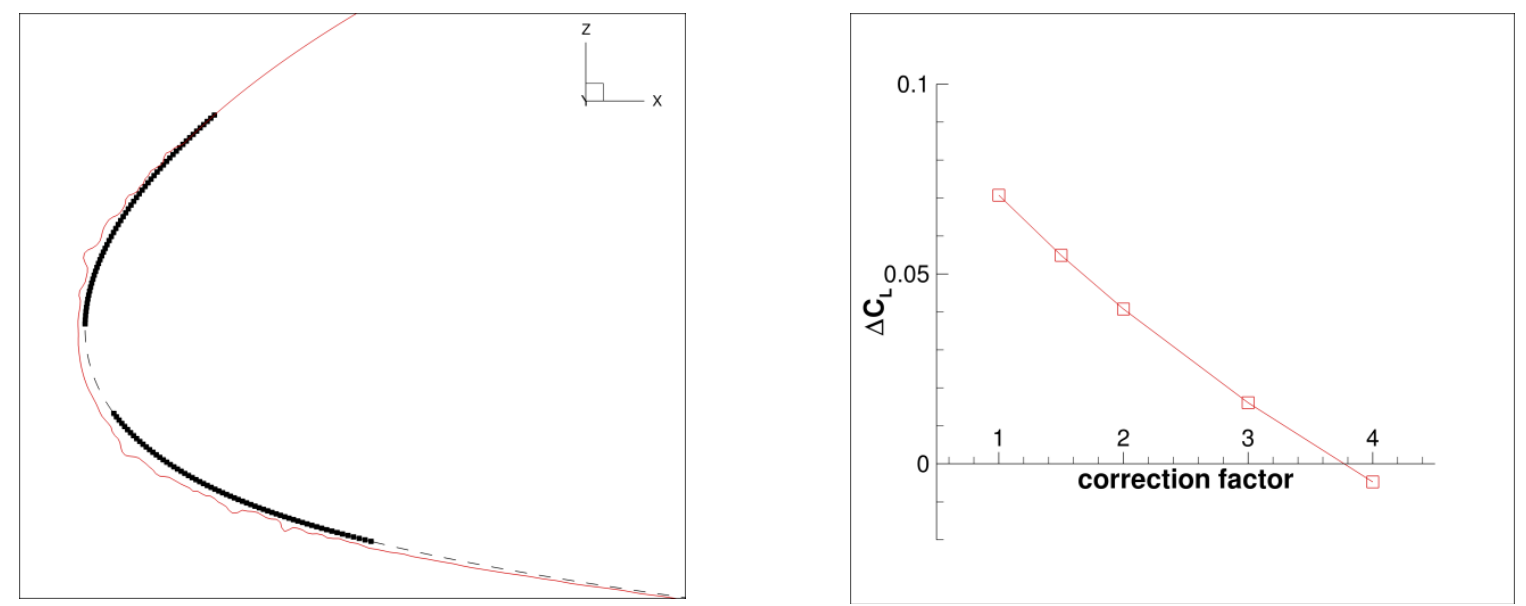

Figure 14: Set-up of roughness model for EG1126. Left: position of roughness model at airfoil leading edge (black symbols); right: difference of lift coefficient between modelled and gridded roughness at $\underline{\alpha=10^{\circ} \text { versus correction factor on ESGH }}$ 


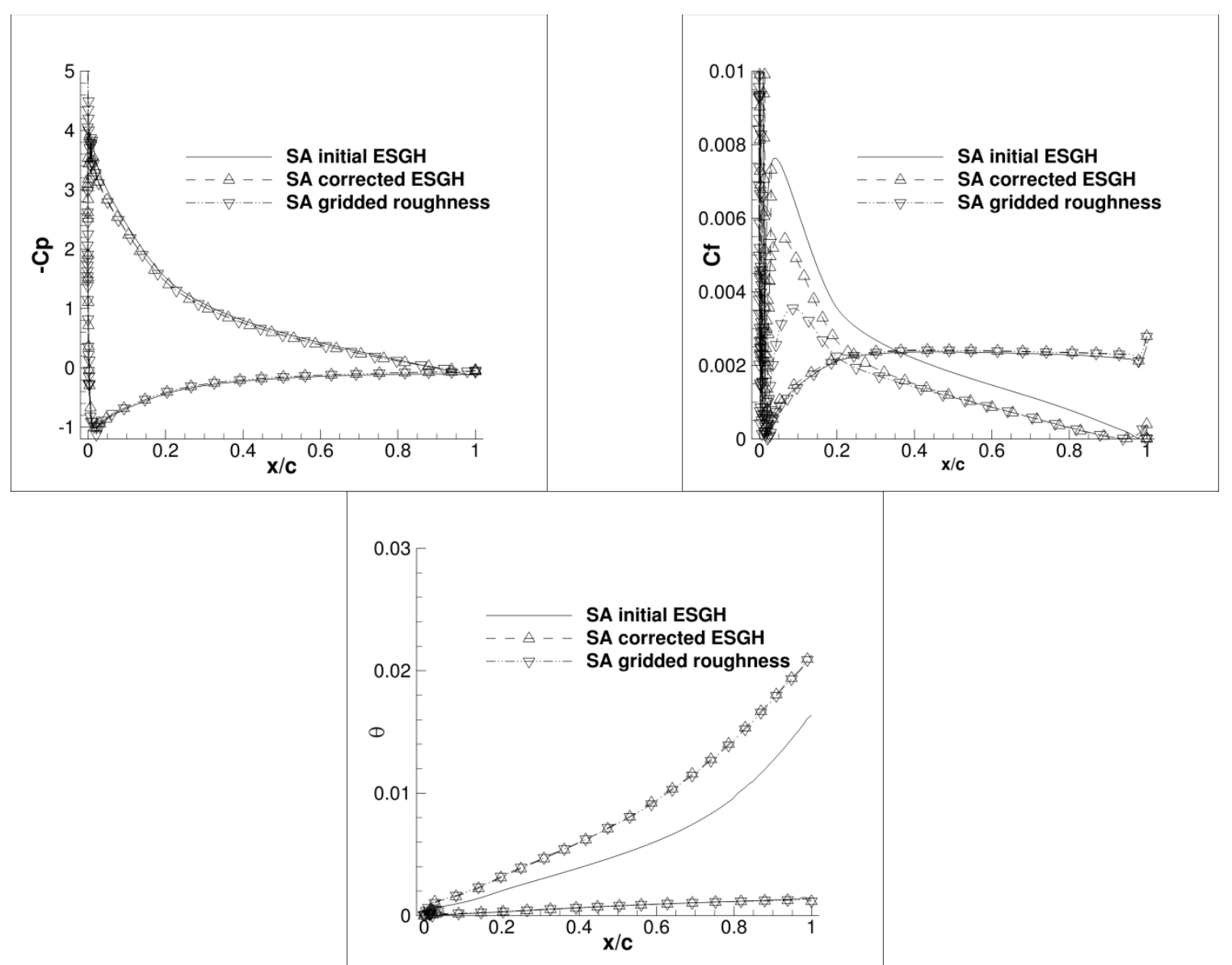

Figure 15: Comparison of chordwise distribution of pressure, skin-friction and momentum thickness for the gridded roughness and the modelled one with initial and corrected ESGH at $\alpha=10^{\circ}$

\section{Convergence of simulations}

Very good convergence to steady-state is reached with the SA model, whatever a gridded or modelled roughness is used. For all incidences computed, more than 9 orders of magnitude residual reduction and good stabilization of the aerodynamic coefficients are obtained. The DRSM SSG/LRR- $\omega$ model also allows satisfactory steady-state convergence of the simulations before stall. The density residual drops by more than 4 orders of magnitude and a good stabilization of the aerodynamic coefficients is obtained, even close to the $C_{\text {Lmax }}$ at $\alpha=12^{\circ}$. At $\alpha=13^{\circ}$, the flow is naturally unsteady with strong vortex shedding and the dual time-stepping method had to be applied. The lift coefficient oscillates with large amplitude due to vortex shedding (Figure 16). For comparison with experimental data, this unsteady solution was time-averaged. 

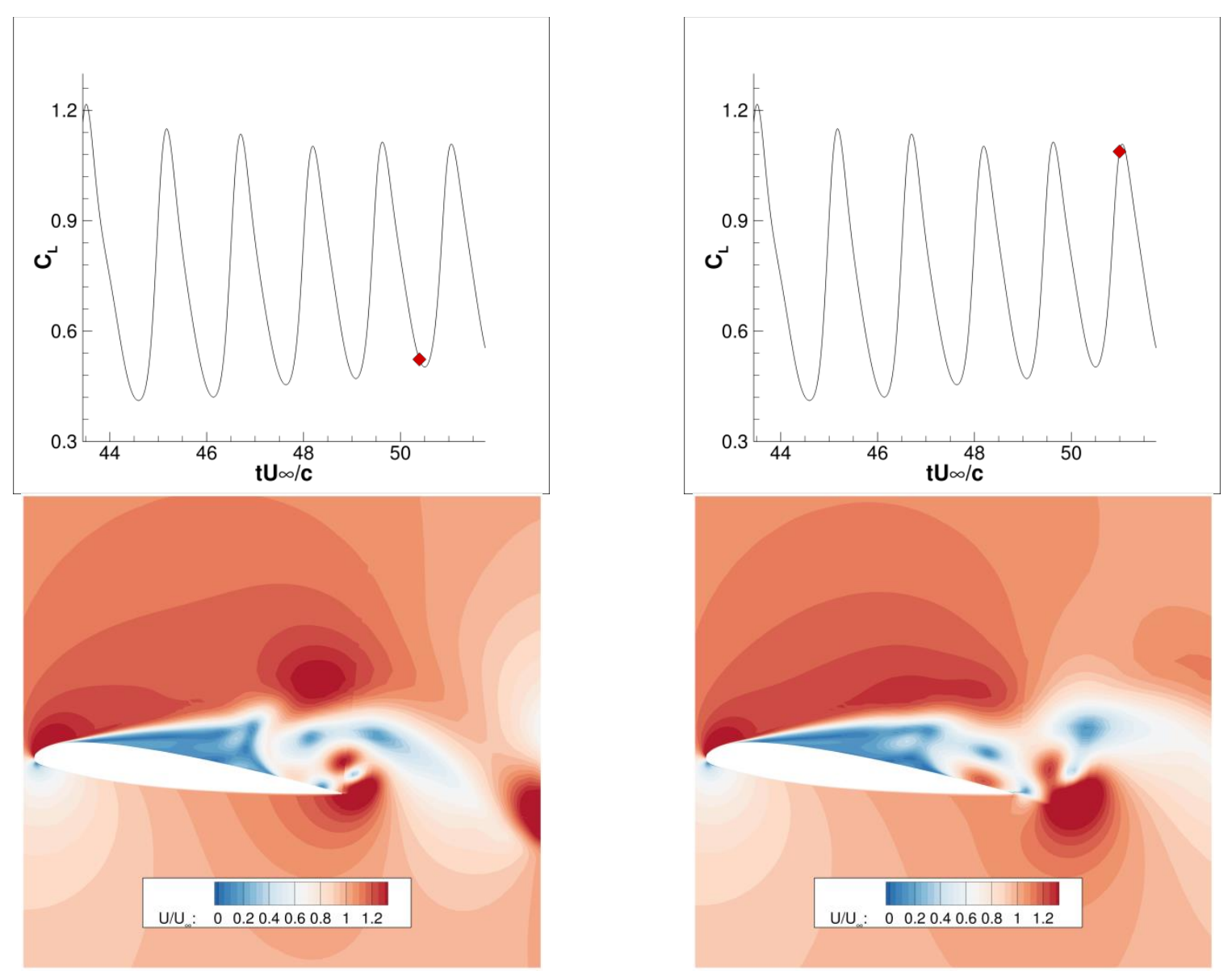

Figure 16: Lift evolution and contour of instantaneous velocity magnitude computed with DRSM at $\alpha=13^{\circ}$ for two times of the simulation depicted by symbols on lift curve

\section{Comparison of solutions}

A comparison of the predicted surface pressure distributions with F1 experiment is shown in Figure 17 for 3 selected angles of attack: $\alpha=8^{\circ} / 12^{\circ} / 13^{\circ}$ and the 3 models: SA and DRSM with gridded roughness and SA with modelled roughness. At $\alpha=8^{\circ}$, the flow is attached and all models give very similar solutions in good agreement with experiment. However, leading edge roughness induce a small pressure plateau around $\mathrm{x} / \mathrm{c}=5 \%$ not captured by the simulation. As shown in Figure 18 , it comes from a small bubble generated by the biggest roughness, which extends further downstream as the angle of attack is increased. Most likely predicting its effect on pressure distribution would require a finer chordwise discretization with respect to the one used in the present work, though this small discrepancy does not affect the good correlation with experiment elsewhere. As the angle of attack is increased, trailing edge separation occurs and progresses towards the leading edge. However, airfoil stall is predicted too late by 
all models. While stall occurs at $\alpha=12^{\circ}$ in the experiment, the simulations only predict limited trailing edge separation for this angle of attack (Figure 19). DRSM is closer to experiment as it predicts flow separation starting from $60 \%$ chord, while it starts around $70 \%$ chord with SA for both the gridded and modelled roughness. The airfoil is stalled at $\alpha=13^{\circ}$ with DRSM, providing reasonably good correlation of the time-averaged pressure with experiment. The main discrepancy comes from the trailing edge where large vortex shedding induces suction not noticeable in the experiment. Maybe the mesh discretization is not sufficiently fine in this region, more especially at the base of the trailing edge where the grid is not adapted to a viscous resolution of the flow. Furthermore, the actual roughness in the tests is $3 \mathrm{D}$ and it is likely that a pure 2D simulation promotes too large vortex shedding. Indeed, three-dimensional simulations presented in [16] clearly show that the flow separation induced by roughness is 3D. Last, the pressure distribution is probably not yet statistically converged for this low-frequency and high-amplitude unsteadiness in the vicinity of the trailing edge, as only eight convective times were considered for averaging. Finally, it can also be noted that the occurrence of stall corresponds to the time when the leading edge recirculation induced by the rough elements connects with the trailing edge separation which has moved sufficiently forward (Figure 20), thus leading to full separation of the upper surface of the airfoil. With SA model stall has not yet occurred at $\alpha=13^{\circ}$ (it only occurs at $\alpha=14^{\circ}$ for the gridded roughness), so that the correlation with experiment is less satisfactory. The modelled roughness also provides the worst results as it underpredicts flow separation even more. 
a) $\alpha=8^{\circ}$

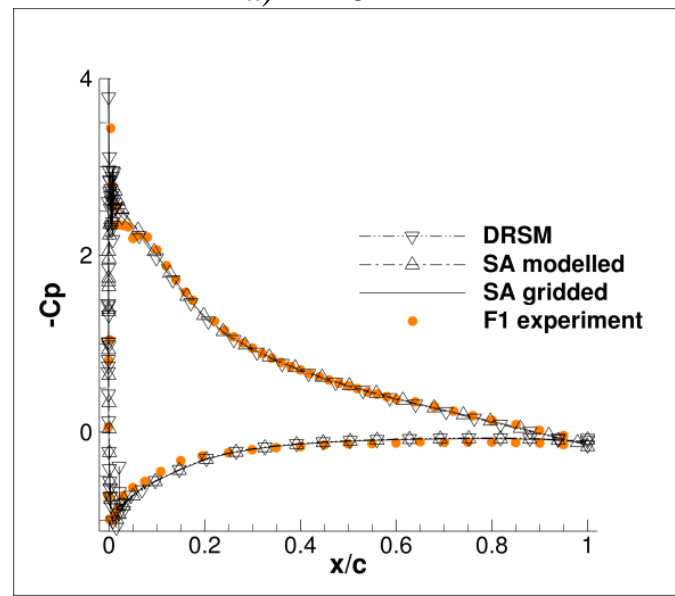

b) $\alpha=12^{\circ}$

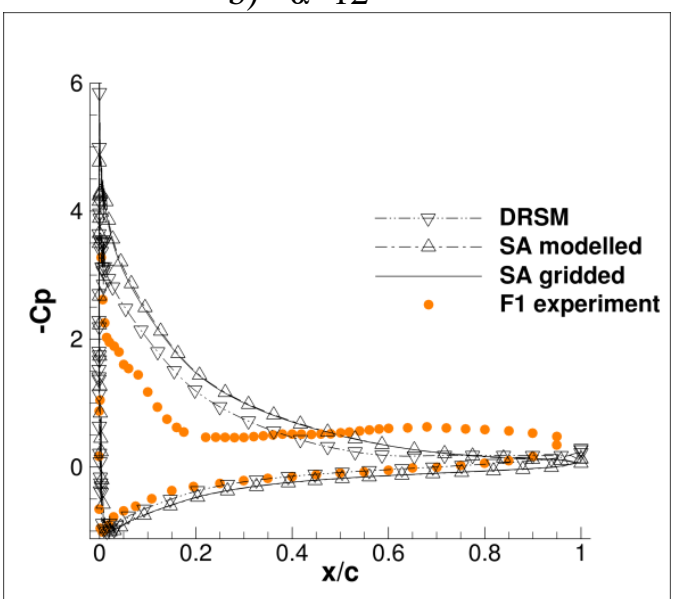

c) $\alpha=13^{\circ}$

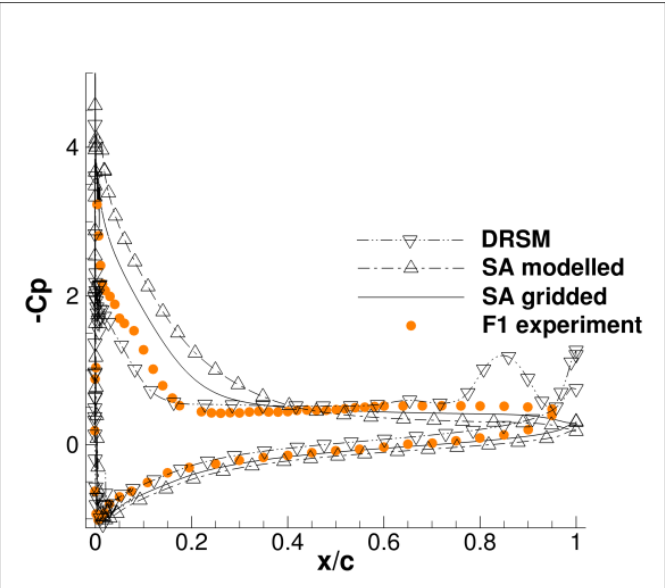

Figure 17: Comparison of chordwise pressure distribution predicted by SA (for gridded and modelled roughness) and DRSM turbulence models for EG1126

a) $\mathrm{SA}$

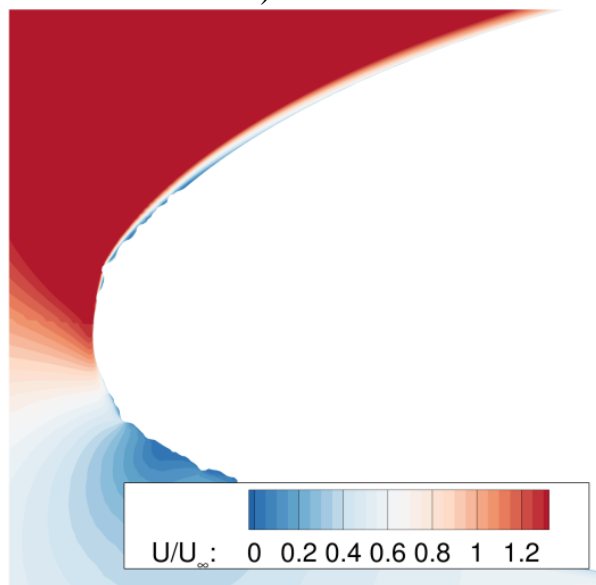

b) DRSM

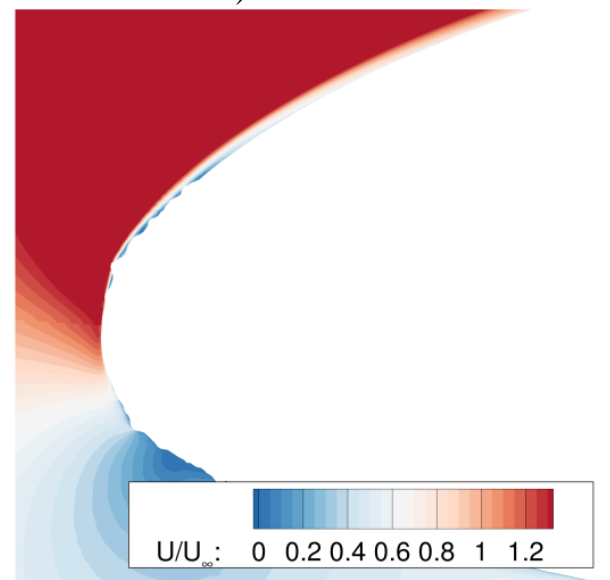

Figure 18: Leading edge detail of velocity magnitude with gridded roughness at $\alpha=8^{\circ}$ 
a) SA

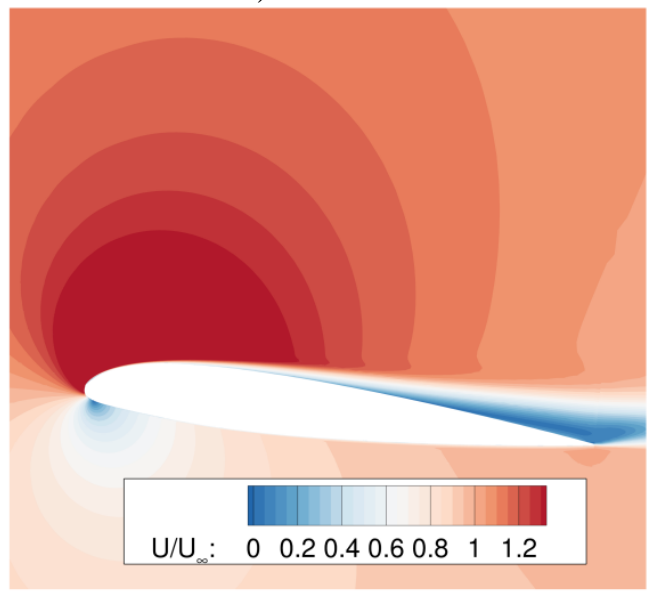

b) DRSM

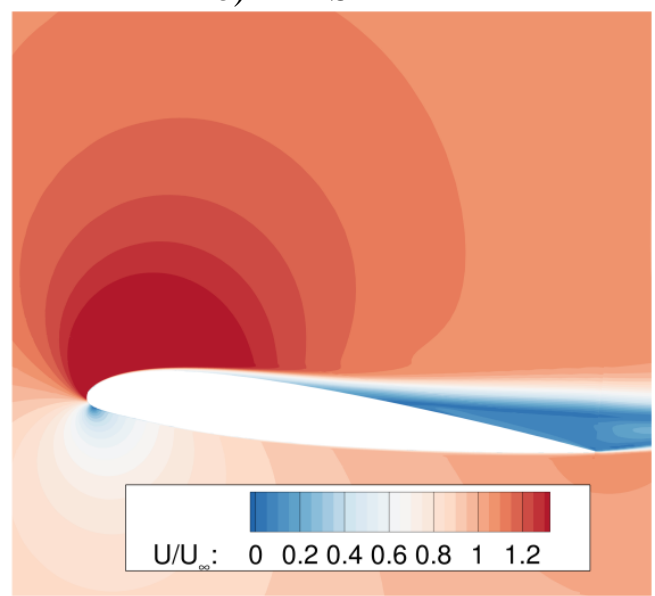

Figure 19: Contour of velocity magnitude with gridded roughness at $\alpha=12^{\circ}$

a) SA

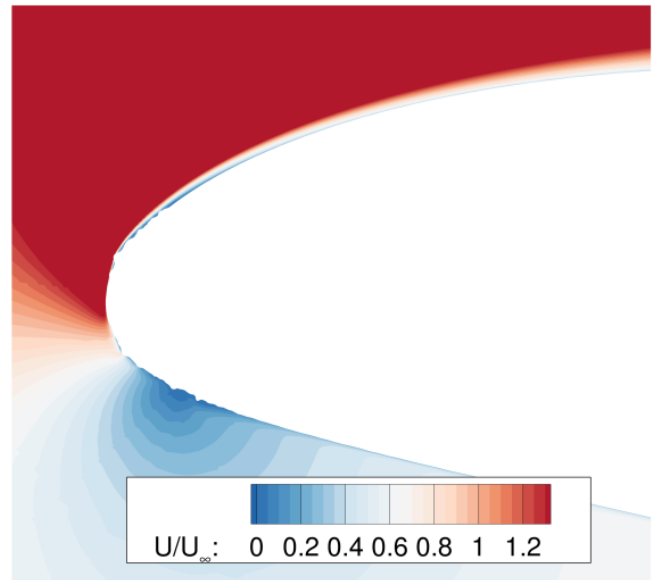

b) DRSM

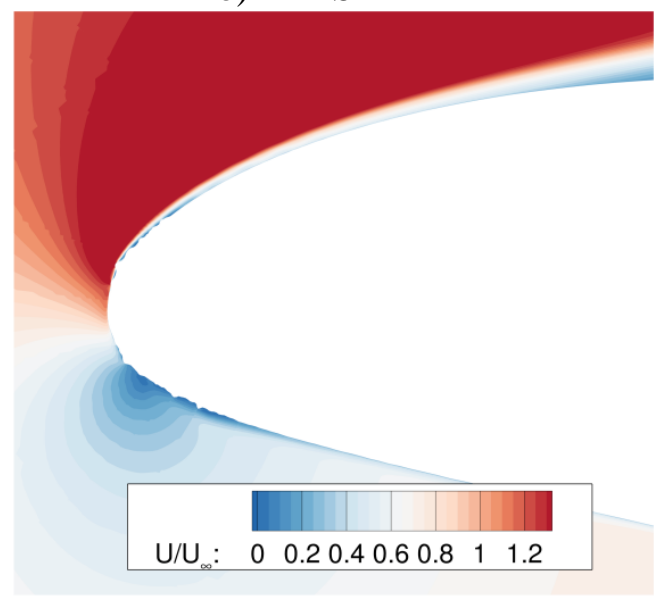

Figure 20: Leading edge detail of velocity magnitude with gridded roughness at $\alpha=13^{\circ}$

\section{Integrated results}

The resulting integrated aerodynamic coefficients are compared with experiment in Figure 21. As mentioned above, the SA results with gridded and modelled roughness match almost perfectly together up to stall. For higher incidences, the gridded roughness predicts more severe stall, consistently with the evolution of pressure distribution. Nevertheless, in both cases the SA model overestimates the $C_{\text {Lmax }}$ of about 0.1 , the stall angle of $1^{\circ}$, and the predicted stall is also too smooth. Before stall, the drag and pitching moment coefficients are fairly well predicted. On the contrary, the DRSM model underestimates the $C_{\text {Lmax }}$ of about the same value 0.1 and the stall region is spread over a range of incidences of $2^{\circ}$ before severe performance degradation with large unsteady content. In the stall region, the pitching moment 
presents an overshoot before abrupt moment stall, not noticeable in the experiment. Again, this may be due to a too coarse discretization of the trailing edge region. Finally, the pitching moment fluctuations in the stalled regime are also too large, and may be attributed to the 2D assumption of the simulation with too strong vortex shedding.
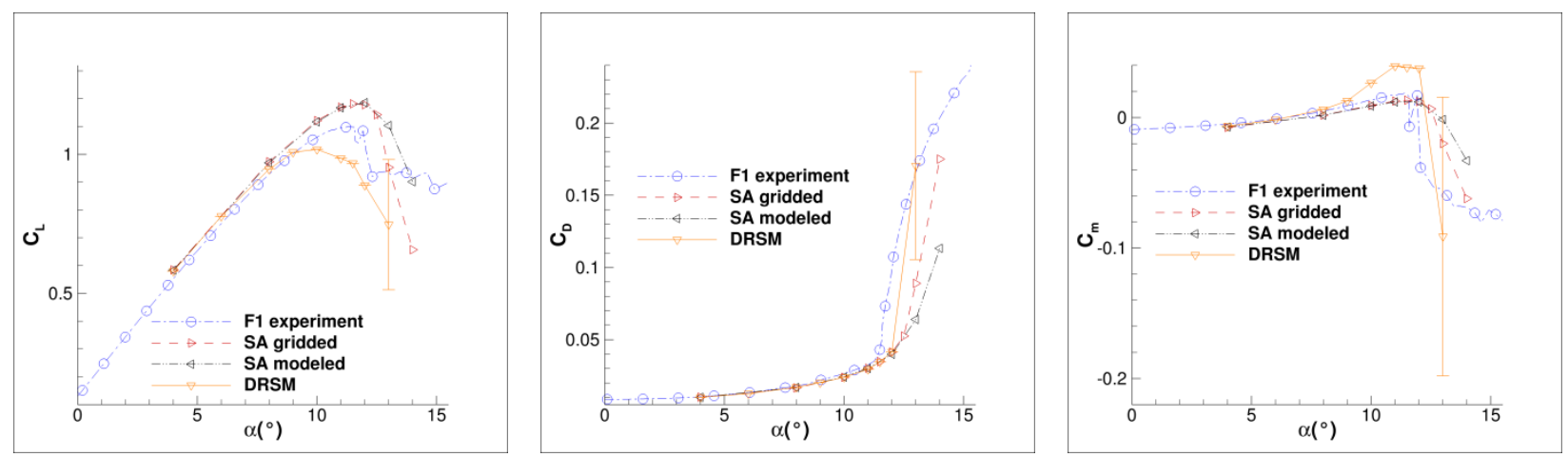

Figure 21: Effect of turbulence model on integrated results for EG1126

\section{Performance degradation due to ice}

A comparison of the polar curves computed for the clean airfoil, the spanwise ridge and the roughness shape with F1 experiment is presented in Figure 22. Two turbulence models are considered, SA and DRSM. In both cases the clean airfoil was computed in fully turbulent mode on the same clean airfoil grid as the one used for applying the roughness model with SA turbulence model. Although the $C_{\text {Lmax }}$ and the stall angle are not perfectly predicted by the present simulations, the ranking of airfoil as well as the magnitude in lift performance penalty is fairly well estimated by both models. With SA, the $C_{\text {Lmax }}$ of the clean airfoil and of the airfoil with roughness is overestimated by about the same amount (of the order of 0.1 ), together with a stall angle overestimated by $1.5^{\circ}$. Conversely, the $C_{\text {Lmax }}$ of the spanwise ridge is underestimated by 0.15 and the stall angle underestimated by $1^{\circ}$, so that the predicted performance degradation for the most severe case is conservative with respect to experiment. The pitching moment stall is also fairly well predicted, as well as the drag rise due to ice accretion. Contrary to the SA model, the DRSM model tends to underestimate the $C_{\text {Lmax }}$ for the three configurations, but the corresponding discrepancy is smaller and the stall angle generally seems to be better predicted. The degradation of performance due to both ice shapes is fairly well predicted in terms of lift and drag. However, too large 
positive pitching moments are predicted before moment stall, and their value in the stalled regime presents very large fluctuations. This seems to be due to the large sensitivity of this parameter to trailing edge flow prediction.
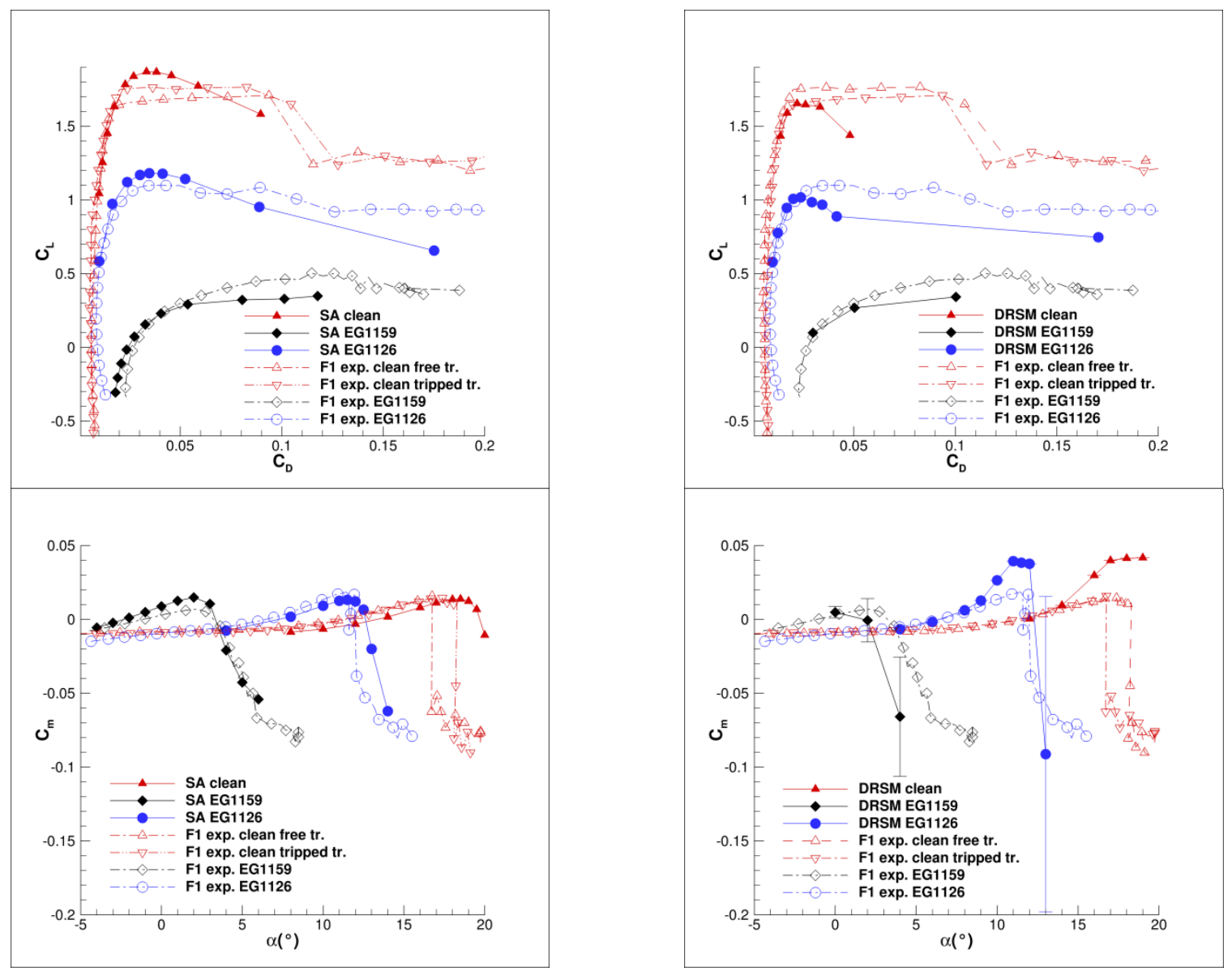

Figure 22. Comparison of polar curves computed by elsA with the F1 experiment

\section{Unsteady results}

As could be checked before, the time-averaged results obtained for EG1159 at $\alpha=2^{\circ}$ with DRSM model and ZDES are close. The unsteady fluctuations computed by both techniques also present similarities which are discussed in this paragraph.

\section{Statistical convergence}

Before showing the unsteady content of the DRSM and ZDES solutions, a brief overview on the statistical convergence of results is presented. This is more particularly important for ZDES results as a 
relatively short period of convective time was computed for averaging. First the effect of duration of averaging for the chordwise pressure distribution is presented in Figure 23 for the DRSM and ZDES II simulations. The ZDES results (Figure $23 \mathrm{~b}$ ) compare the mean pressure coefficient computed after 3.17 convective times and 4.07 convective times, showing only small differences on the upper surface of the airfoil downstream the recirculation bubble where strong vortex shedding is obtained. For DRSM (Figure 23 a), the mean solutions are compared after 6.20 and 9.16 convective times. Although a longer period of time was computed, small differences can yet be noted on the upper surface of the airfoil aft of the spanwise ridge, again due to the strong vortex shedding downstream the recirculation. This is indeed a low-frequency phenomenon very demanding for being perfectly statistically converged, so that lower frequency phenomena discussed below will have to be considered with care. In both cases, the pressure distributions on the lower surface of the airfoil and upstream the recirculation on the upper surface are identical. Therefore, as the mean pressure variations observed remain quite small, the comparisons with experiment and with other turbulence models presented in the paper are meaningful.

a) DRSM

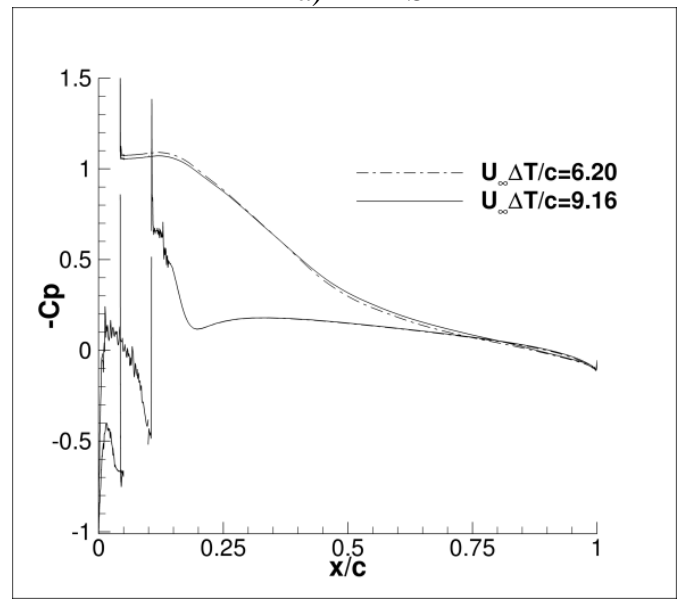

Figure 23: Effect of duration of time-averaging on mean pressure coefficient

b) ZDES II

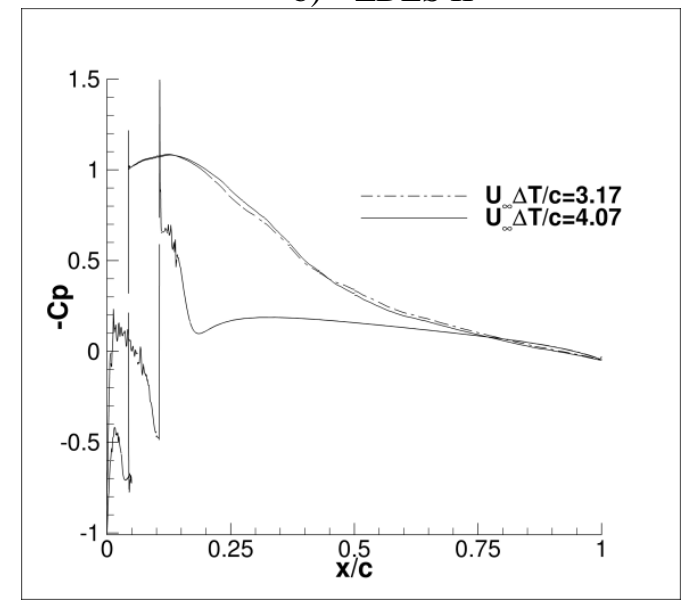

As far as RMS quantities are concerned, only ZDES results are presented as we are interested in the Reynolds stresses which are modelled by DRSM and therefore do no need to be time-averaged to be significant. The ZDES II results are plotted in Figure 24 and Figure 25 for the longitudinal normal stress and the shear stress with a period of time-averaging varying from 1.36 to 4.07 convective time units. Although the contours get smoother as the time interval is increased, the computed Reynolds stresses 
weakly depend on the convective time used for averaging. The other normal stresses also show a similar convergence behavior, thus proving that reasonable statistical convergence is rapidly obtained for the Reynolds stress tensor. This result is consistent with a characteristic integral time scale of turbulence in the free-shear layer $\left(\delta_{\omega} /\left(U_{u}-U_{l}\right)\right)$ being much smaller than the convective time unit $\left(c / U_{\infty}\right)$. In addition, because the mean flow is two-dimensional, the results are also averaged along the spanwise direction over a large number of values thus explaining this fast convergence of statistical results.

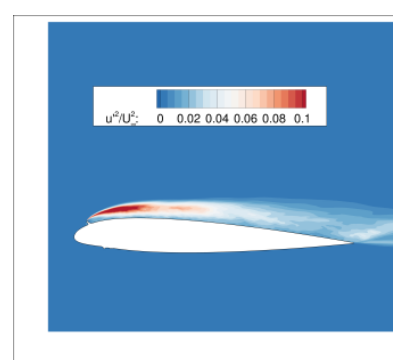

$\mathrm{U}_{\infty} \Delta \mathrm{T} / \mathrm{c}=1.36$

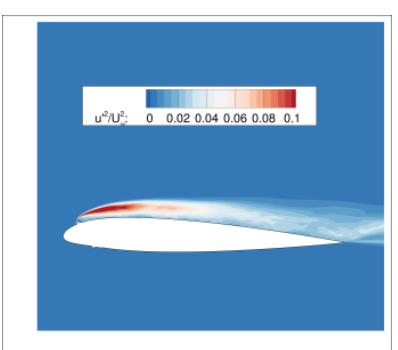

$\mathrm{U}_{\infty} \Delta \mathrm{T} / \mathrm{c}=2.26$

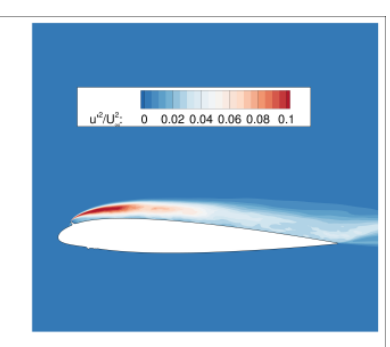

$\mathrm{U}_{\infty} \Delta \mathrm{T} / \mathrm{c}=3.17$

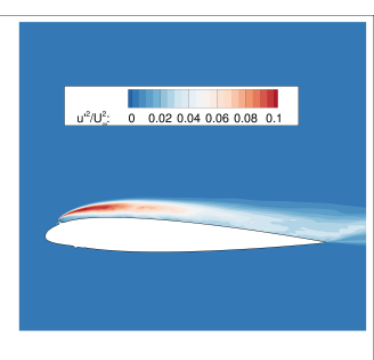

$\mathrm{U}_{\infty} \Delta \mathrm{T} / \mathrm{c}=4.07$

Figure 24: Effect of duration of time averaging on contour of $\overline{\boldsymbol{u}^{\prime 2}}$

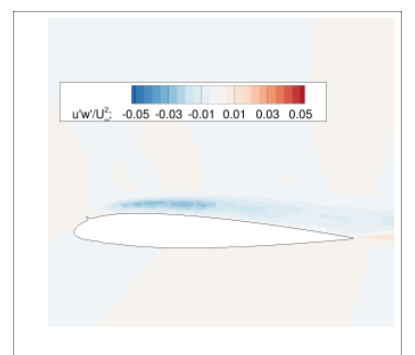

$\mathrm{U}_{\infty} \Delta \mathrm{T} / \mathrm{c}=1.36$

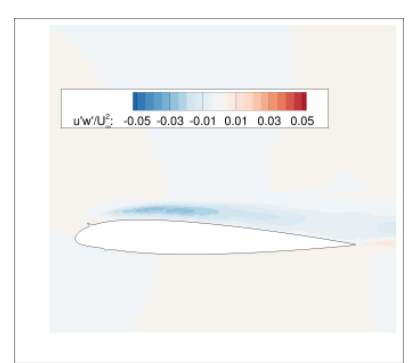

$\mathrm{U}_{\infty} \Delta \mathrm{T} / \mathrm{c}=2.26$

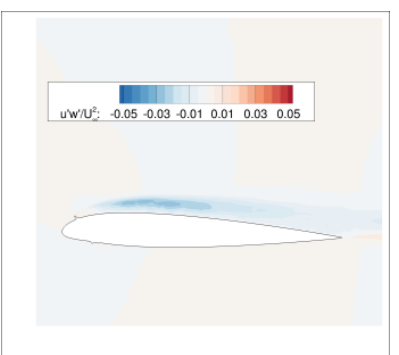

$\mathrm{U}_{\infty} \Delta \mathrm{T} / \mathrm{c}=3.17$

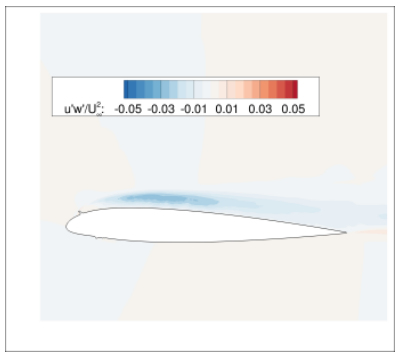

$\mathrm{U}_{\infty} \Delta \mathrm{T} / \mathrm{c}=4.07$

Figure 25: Effect of duration of time averaging on contour of $\overline{u^{\prime} w^{\prime}}$

\section{Unsteady loads}

The time evolution of lift exhibits a low frequency and high amplitude oscillation in the unsteady computations corresponding to vortex shedding downstream the recirculation bubble. This periodic vortex shedding can be observed for the three simulations in Figure 26 from the high-velocity spots above the airfoil. The period of the shedding can be estimated by spectral analysis of the lift, drag and pitching moment oscillations (see Figure 27 for the lift spectra, where the identified shedding frequency is indicated by the dotted line). It corresponds to a Strouhal number, based on airfoil chord and freestream velocity, of $1.90 \pm 0.32$ for DRSM, of $1.67 \pm 0.23$ for ZDES I and of $1.61 \pm 0.14$ for ZDES II. The 
corresponding frequencies are equal to $71.8 \pm 12.1 \mathrm{~Hz}, 63.1 \pm 8.6 \mathrm{~Hz}$ and $60.6 \pm 5.3 \mathrm{~Hz}$ respectively for a Mach number $M_{\infty}=0.2$ and a chord $c=1.829 \mathrm{~m}$. These estimates show that the shedding frequency is in the same range for the 3 computations, thus providing a qualitative agreement between ZDES and DRSM. As mentioned before, a longer period of computed time would be necessary to get a more quantitative comparison of the vortex shedding phenomena between the models.

a) ZDES mesh I

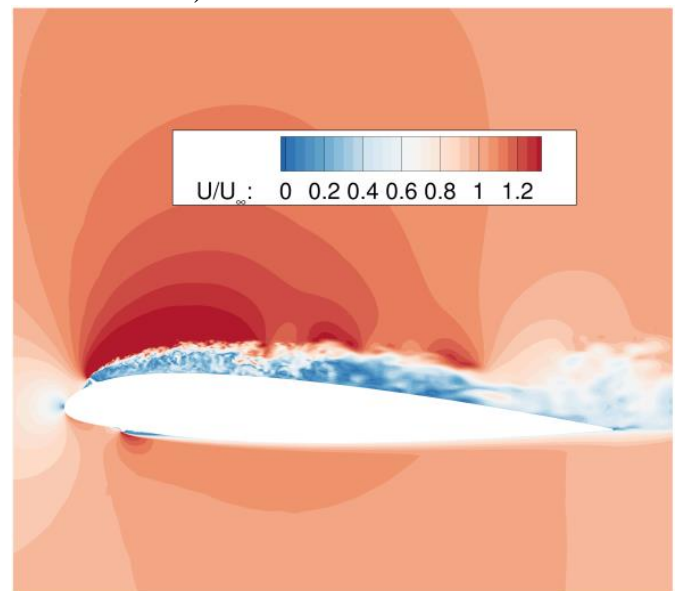

b) ZDES mesh II

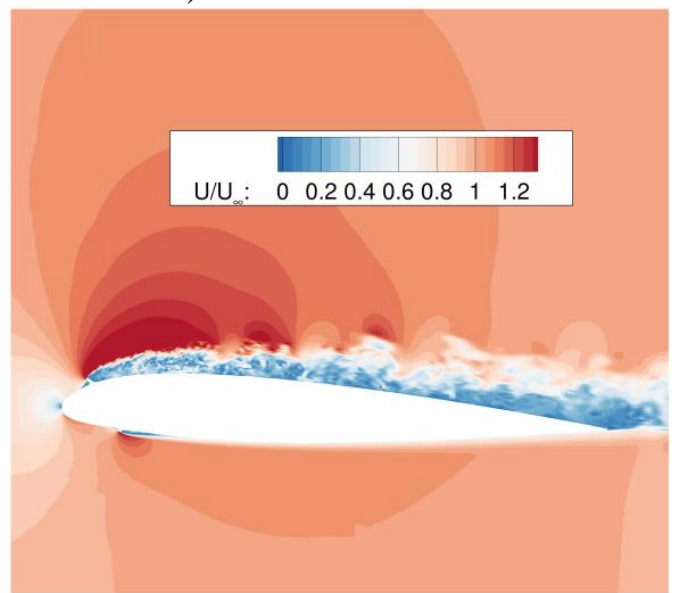

c) DRSM SSG- $\omega$

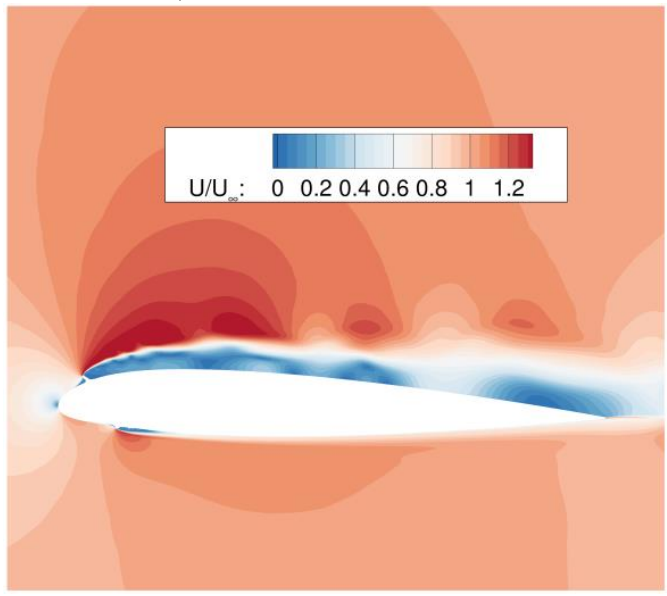

Figure 26: Example of instantaneous velocity field computed by unsteady DRSM and ZDES simulations 
a) DRSM

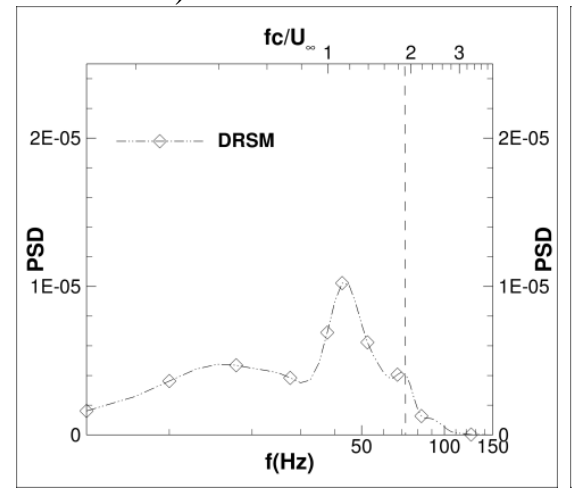

Figure 27: PSD of lift coefficient computed b) ZDES Mesh I

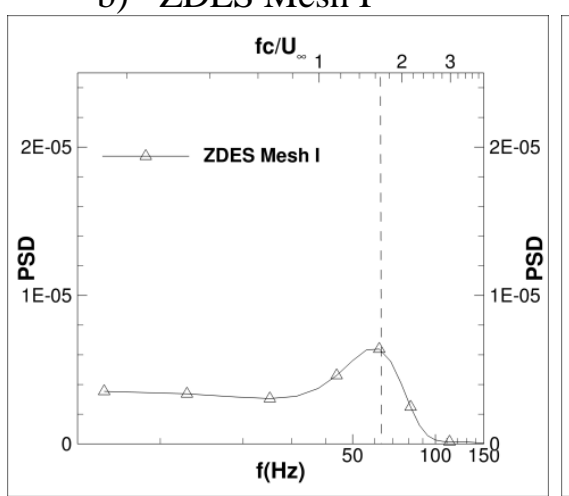

c) ZDES Mesh II

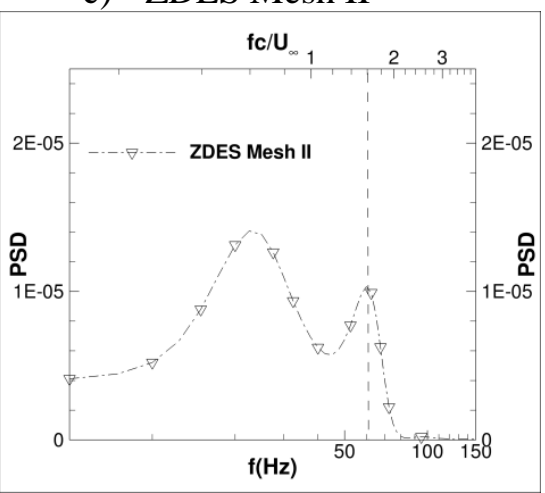

shedding frequency

\section{Reynolds stresses}

The Reynolds stress contours of the time-averaged DRSM solution are compared to their spanwiseaveraged counterparts computed by ZDES I and II in Figure 28 to Figure 30. For ZDES, the subgrid part of the Reynolds stress tensor is neglected as only the large scale fluctuations coming from the unsteady content of the solution are taken into account. This means that the lower surface stresses computed by ZDES I and II are not relevant. On the upper surface, very similar levels are obtained in the three simulations for all four meaningful components of the Reynolds stresses, which all use the same contours scale with a simple shift for the shear stress as it includes both positive and negative values. This means that the most energetic stresses, coming from the larger scales, are captured by ZDES and match those modelled by DRSM. As far as normal stresses are concerned, the largest one is the longitudinal component $\overline{u^{\prime 2}}$. It is mainly localized in the shear layer at the edge of the recirculation and downstream within the vortex shedding area. The maximum level is very similar between the three computations, altough it seems to be localized slightly more upstream in ZDES than in DRSM. The other two normal stresses have about the same magnitude with a maximum value of around 50\% that of the longitudinal stress, and the spanwise component $\overline{v^{\prime 2}}$ is a little larger than the vertical one $\overline{w^{\prime 2}}$, especially right downstream the spanwise ridge. Finally the only meaningful shear stress $\overline{u^{\prime} w^{\prime}}$ is mainly negative, with a maximum value slightly lower than that of the normal stresses $\overline{v^{\prime 2}}$ and $\overline{w^{\prime 2}}$ and a localization in the shear layer too. It is also interesting to note that the ratios between the components of the Reynolds stress tensor 
and the turbulent kinetic energy are of the same order of magnitude as the classical values obtained for homogeneous turbulent shear flows (Figure 31: ratio of 1.1 for $\overline{u^{\prime 2}}, 0.6$ for $\overline{v^{\prime 2}}, 0.4$ for $\overline{w^{\prime 2}}$, and -0.3 for $\left.\overline{u^{\prime} w^{\prime}}\right)$. Right downstream the ridge, a small region far from equilibrium can yet be noted with a balance of the Reynolds stresses clearly off the distribution downstream.

a) $\overline{u^{\prime 2}}$

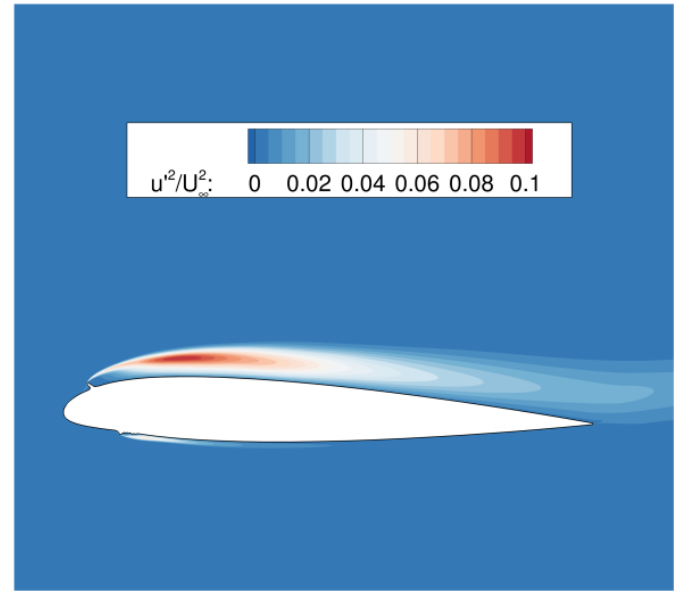

c) $\overline{w^{\prime 2}}$

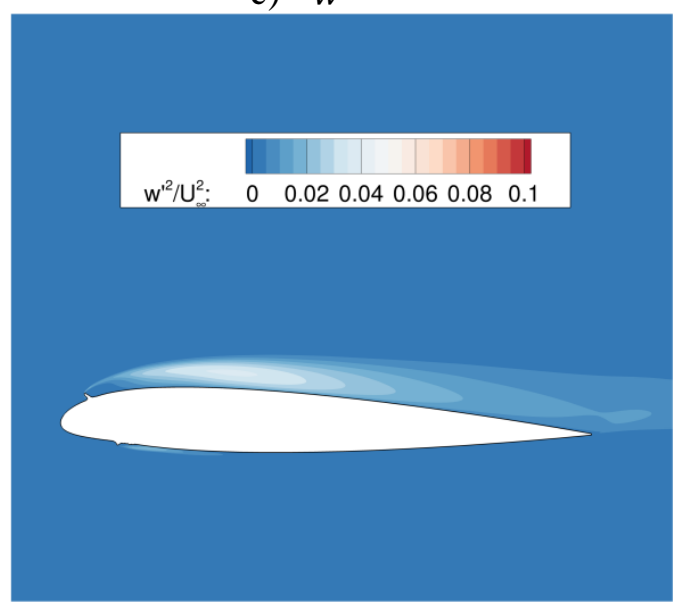

b) $\overline{v^{\prime 2}}$

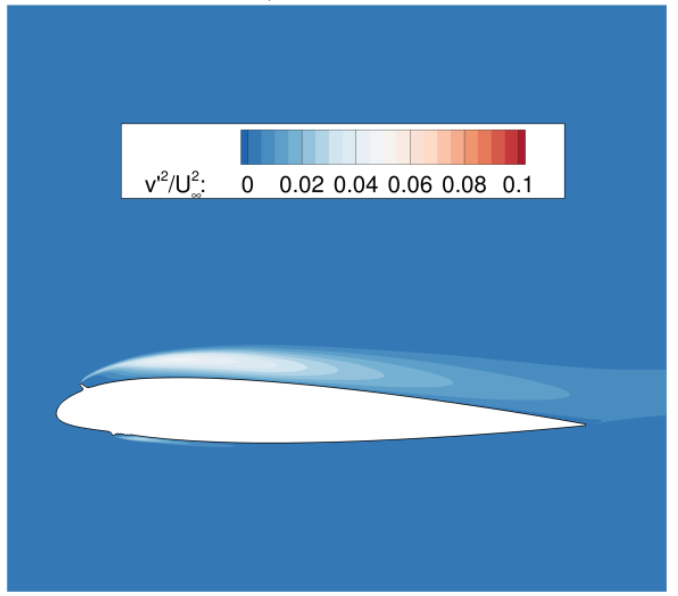

d) $\overline{u^{\prime} w^{\prime}}$
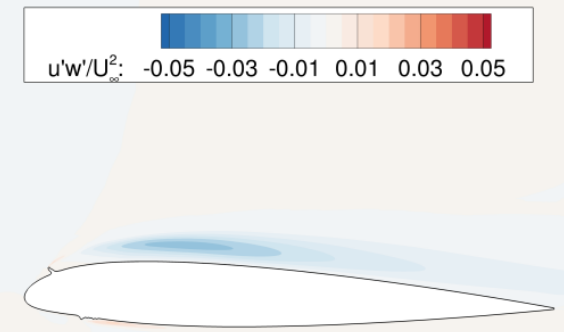

Figure 28: Contours of Reynolds-stress components computed by DRSM for EG1159 at $\alpha=2^{\circ}$ 
a) $\overline{u^{\prime 2}}$

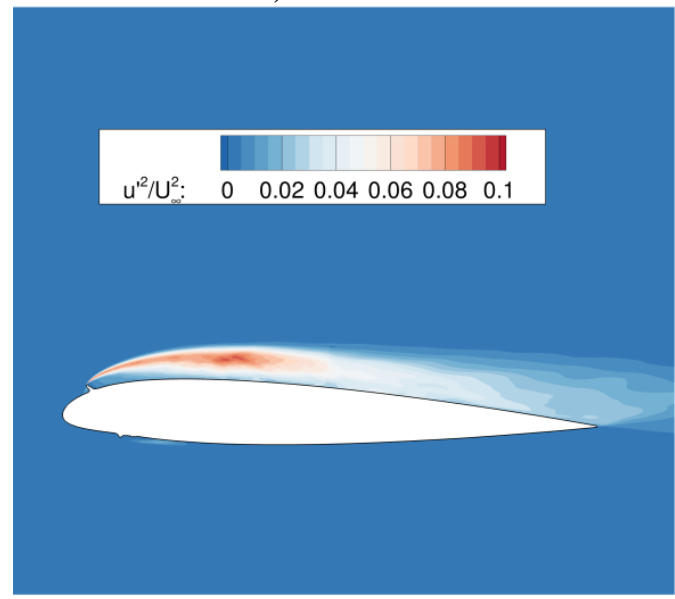

c) $\overline{w^{\prime 2}}$

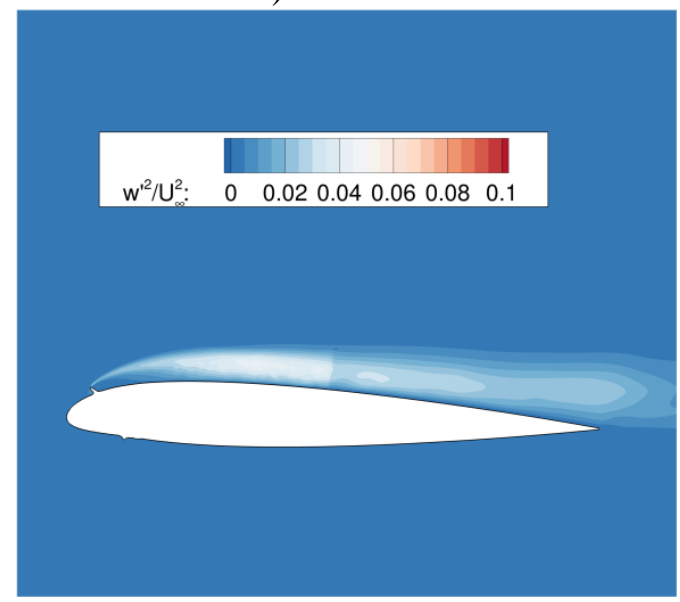

b) $\overline{v^{\prime 2}}$

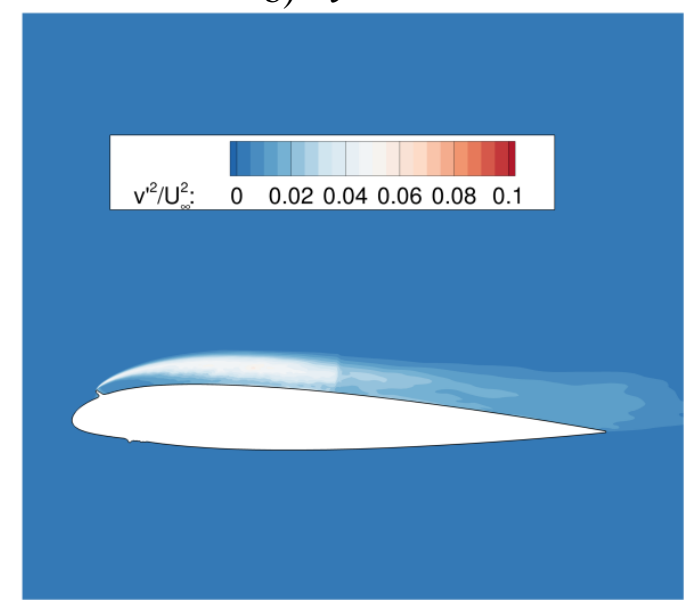

d) $\overline{u^{\prime} w^{\prime}}$
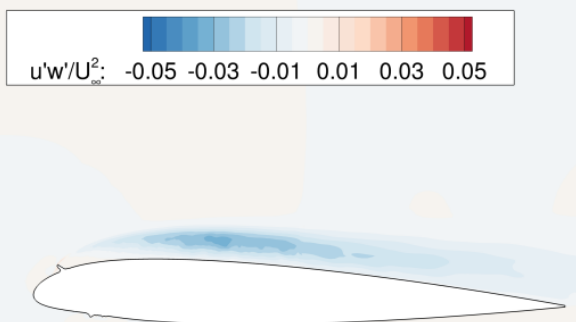

Figure 29: Contours of reconstructed Reynolds-stress components from ZDES I for EG1159 at $\alpha=2^{\circ}$ 
a) $\overline{u^{\prime 2}}$

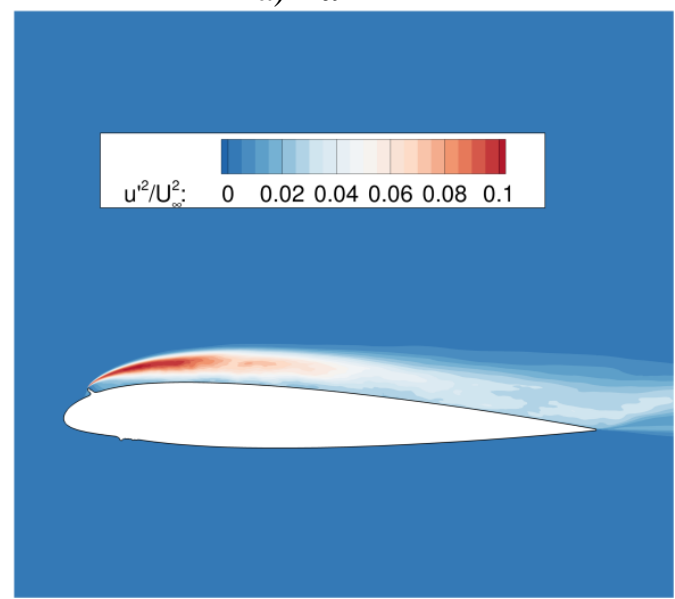

c) $\overline{w^{\prime 2}}$

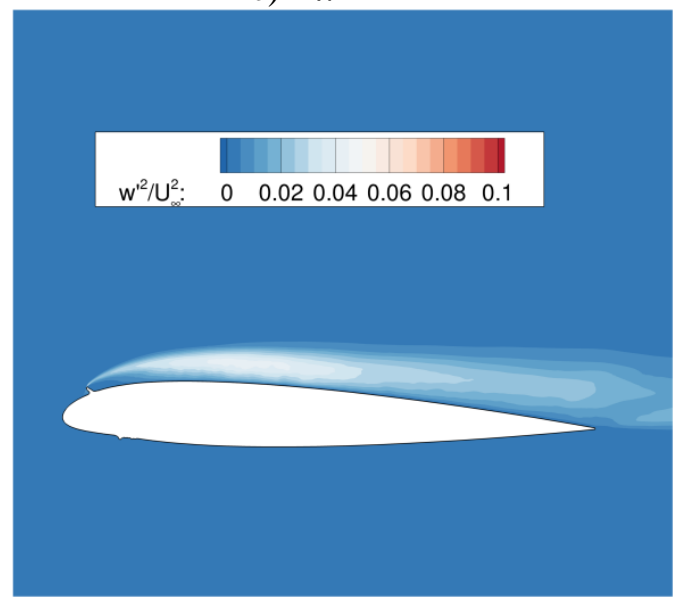

b) $\overline{v^{\prime 2}}$

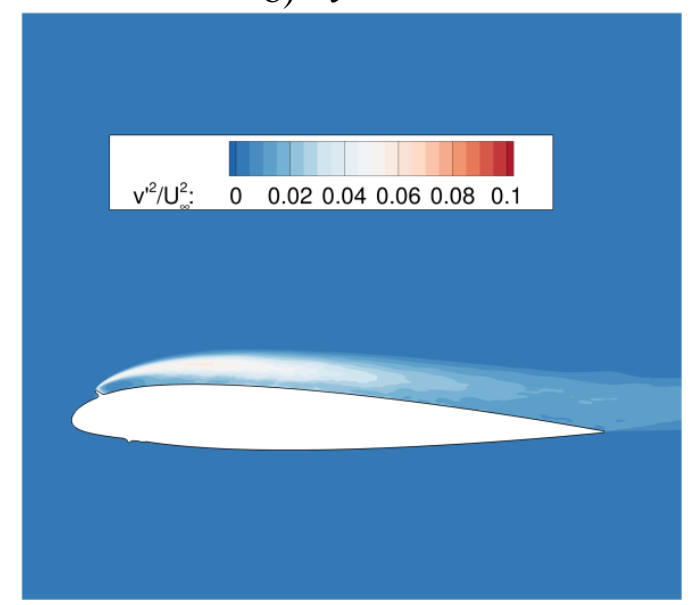

d) $\overline{u^{\prime} w^{\prime}}$
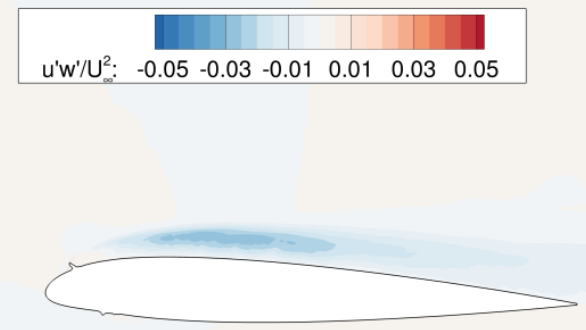

Figure 30: Contours of reconstructed Reynolds-stress components from ZDES II for EG1159 at $\alpha=2^{\circ}$ 
a) $\overline{u^{\prime 2}} / k$

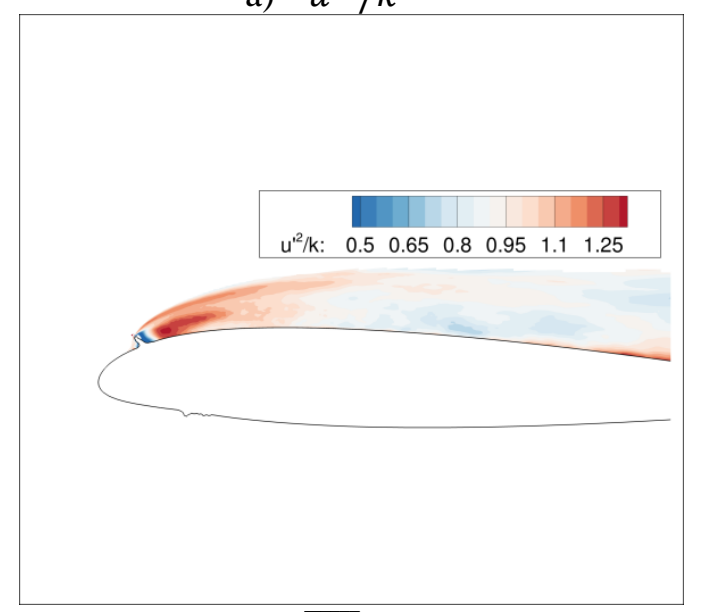

c) $\overline{w^{\prime 2}} / k$

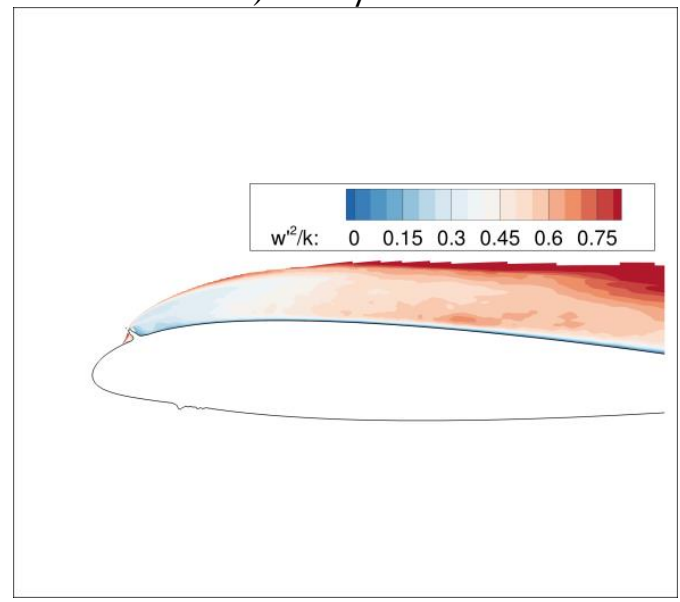

b) $\overline{v^{\prime 2}} / k$

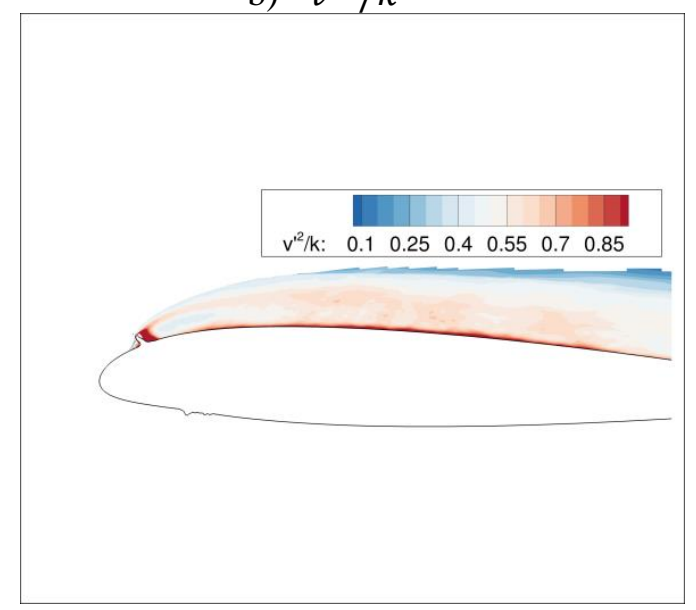

d) $\overline{u^{\prime} w^{\prime}} / k$

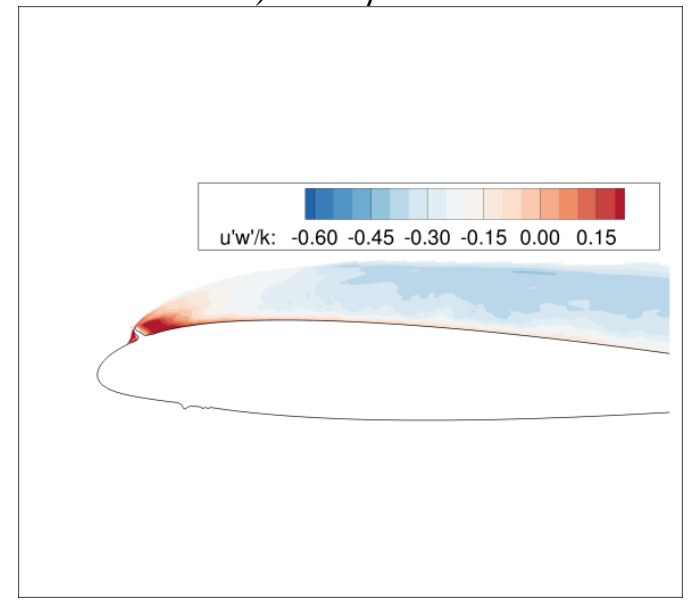

Figure 31: Contours of reconstructed Reynolds-stress components normalized by turbulent kinetic energy from ZDES II for EG1159 at $\alpha=2^{\circ}$

\section{Pressure spectra}

Higher frequency content is also present in the solutions, mainly in the ZDES simulations. Probes were set in the computational domain in order to record the time evolution of the field variables. The spectra of the pressure fluctuations are plotted for several locations along the shear layer and close to the wall in the recirculation bubble. They were computed using Welch method [37] with Hamming window and 50\% overlap, with an averaging over at least 10 blocks. The Power Spectral Density (PSD) of pressure fluctuation $\mathrm{G}(\mathrm{f})$ is plotted in red in $\log$-log scales, while its normalized counterpart $f . G(f) / \sigma^{2}$ is plotted in blue in linear-log scales, with $\sigma^{2}=\int_{0}^{\infty} G(f) d f=\int_{-\infty}^{\infty} f G(f) d \log (f)$. This second plot thus directly gives the contribution of each frequency band to the RMS pressure fluctuations. 
The first region of interest is the shear layer, with probes located at 5\%,8\% and 24\% chord (Figure 32, Figure 33 and Figure 34). The two ZDES simulations give very similar spectra for all probes (Figure 32 to Figure $34 \mathrm{~d}$ and e). At the first location (Figure 32), right downstream the upper edge of the ridge, they show high-frequency content in the range $1000-30000 \mathrm{~Hz}$, or equivalently Strouhal numbers in the range 30-800. It corresponds to the turbulent fluctuations due to the large scales captured in the simulations, confirming that the destabilization of the shear layer is very fast in the simulations. As expected, the spectra show a widening of the high-frequency fluctuations towards lower frequencies when moving downstream (Figure 33, Figure 34), because of the thickening of the shear layer. This range of frequencies is modelled by DRSM and therefore it cannot appear in the captured spectra (Figure 32 to Figure 34 c). However, a few low-frequency isolated peaks can be observed in the DRSM spectra. Right donwstream the spanwise ridge (Figure 32), a first peak at a Strouhal number (based on airfoil chord and freestream velocity) of the order of 2 can be noted. It corresponds to the vortex shedding frequency which is also noticeable in the unsteady loads. It does not appear in the ZDES spectra, probably because a too short period of time was computed in this case. This frequency is clearly out of the range of turbulent fluctuations captured by ZDES, showing that scale-separation is valid in this case. At this location the DRSM model also predicts a set of higher frequency peaks. When normalized by the local vorticity thickness instead of airfoil chord, estimated at $2.42 \mathrm{~mm}$ here, the corresponding Strouhal numbers are equal to $S t_{\delta_{\omega}}=0.0168 / 0.0323 / 0.0505 / 0.0830$. The approximately integer ratio of frequencies suggests that they all are subharmonics of the same low-frequency motion of the shear layer and of the recirculation. A few isolated peaks also get out of the turbulent spectra of ZDES in the range $S t_{\delta_{\omega}}=$ $0.077-0.156$ which may also be attributed to the same phenomena. In this case, scale-separation does not apply anymore. Further downstream (Figure 33, Figure 34), the DRSM simulation only predicts one pressure peak, the frequency of which decreases along the chord. The ZDES simulations also predict more spreaded peaks in the same requency range. The Strouhal number based on local vorticity thickness is equal to $S t_{\delta_{\omega}}=0.169-0.192$ at $8 \%$ chord and to $S t_{\delta_{\omega}}=0.127-0.137$ at $24 \%$ chord. This 
frequency range is typical of Kelvin-Helmholtz instabilities [38] [39] and thus corresponds to the rolling up of the shear layer prior to vortex shedding. These peaks are at the very lowest frequencies of the turbulent spectrum and therefore scale separation does not hold for this part of the flow where the validity of URANS may become questionable.

a) DRSM

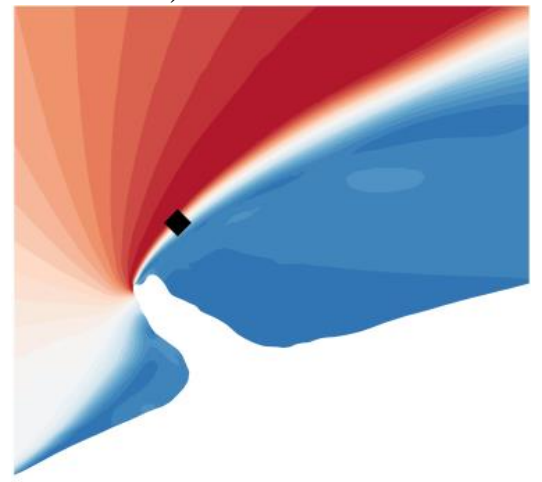

c) RANS spectrum

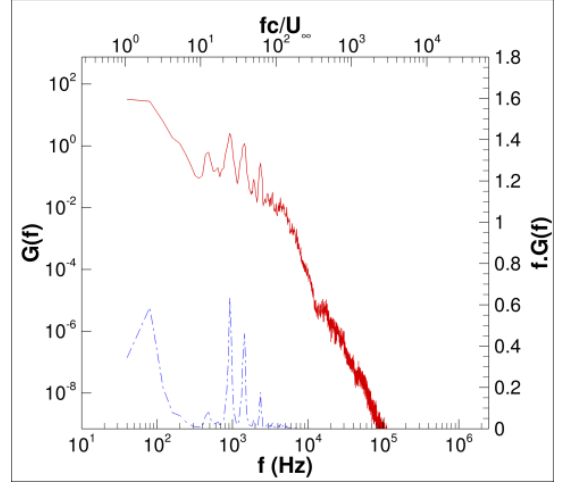

b) ZDES

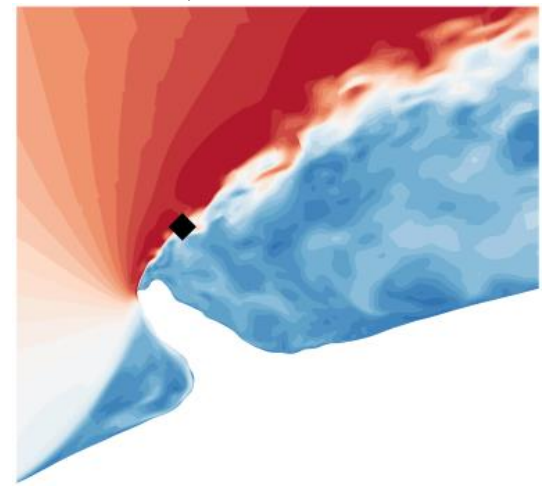

d) Mesh I spectrum

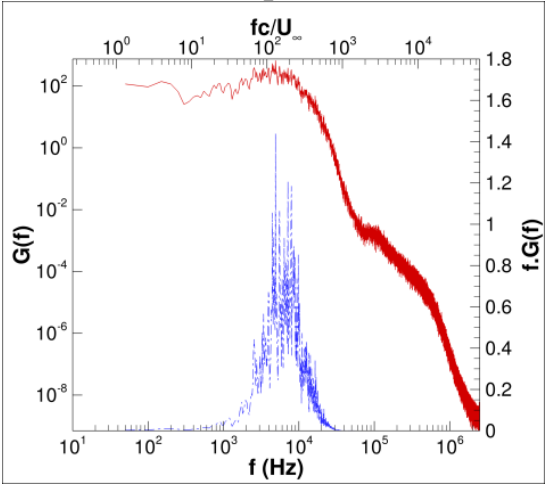

e) Mesh II spectrum

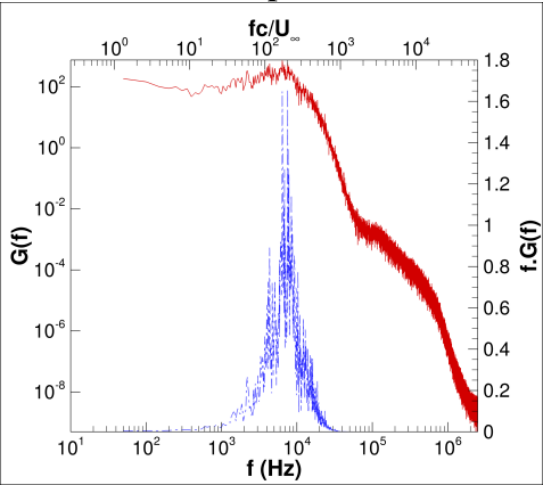

Figure 32: Pressure spectra in the shear layer at 5\% chord - top row: position of probe with velocity contours; bottom row: spectra for DRSM (left) and ZDES mesh I (middle) and II (right) 
a) DRSM

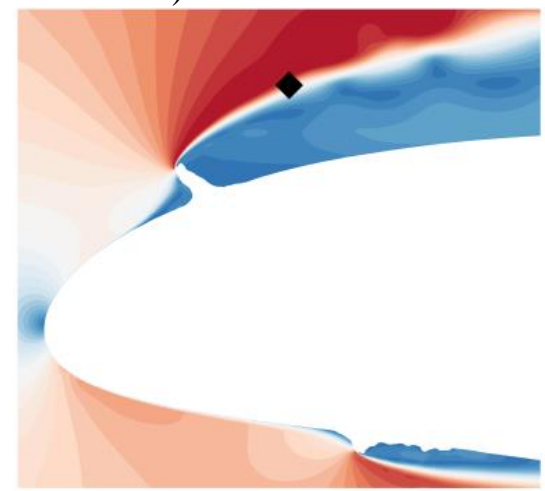

c) RANS spectrum

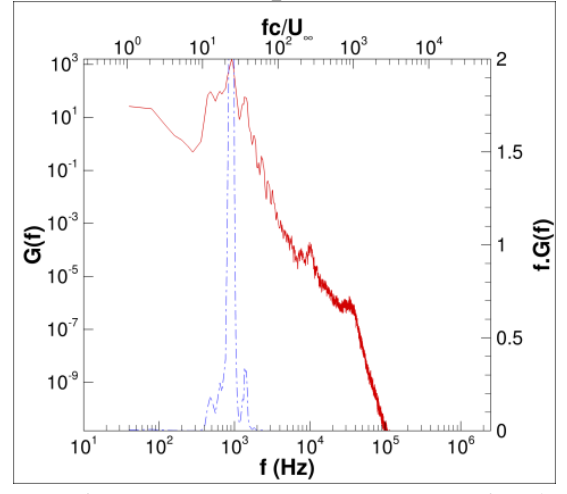

b) ZDES

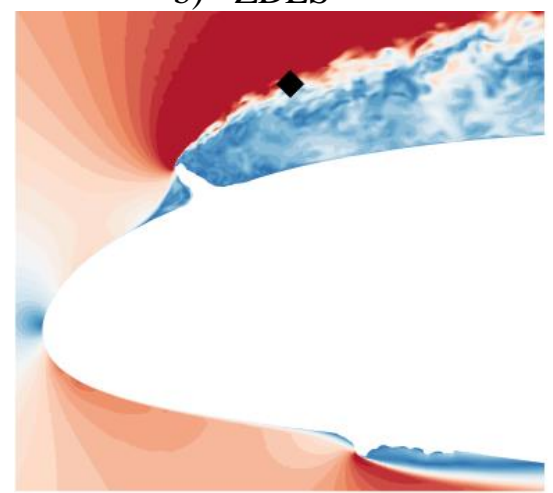

e) Mesh II spectrum

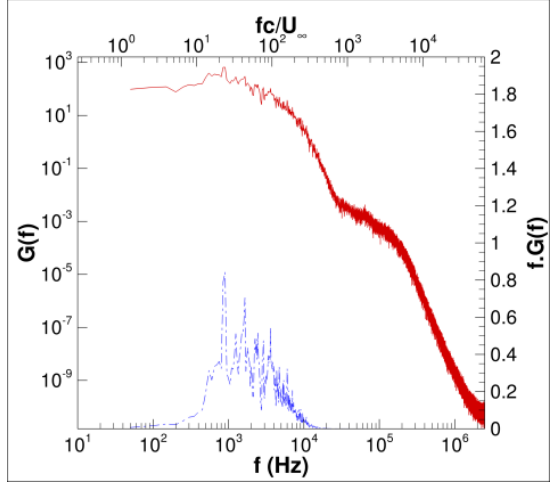

Figure 33: Pressure spectra in the shear layer at $8 \%$ chord - top row: position of probe with velocity contours; bottom row: spectra for DRSM (left) and ZDES mesh I (middle) and II (right) 
a) DRSM

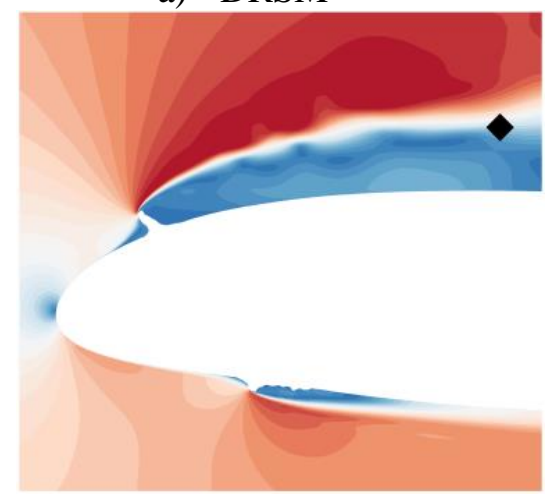

c) RANS spectrum

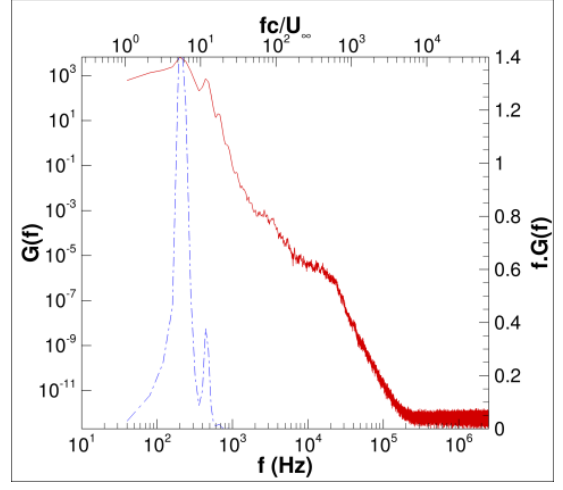

b) ZDES

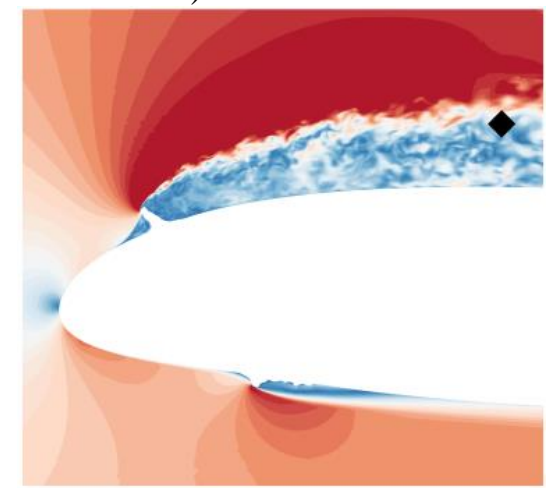

d) Mesh I spectrum

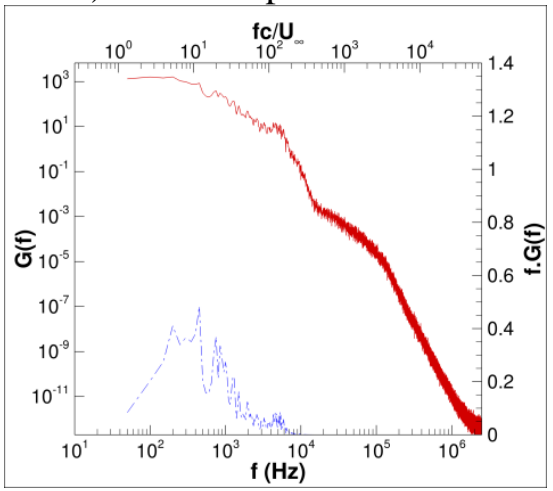

e) Mesh II spectrum

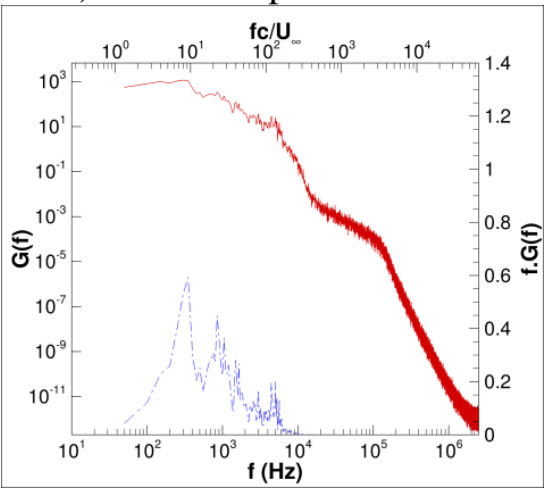

Figure 34: Pressure spectra in the shear layer at $24 \%$ chord - top row: position of probe with velocity contours; bottom row: spectra for DRSM (left) and ZDES mesh I (middle) and II (right)

The pressure spectra near the airfoil wall is now considered for two points located at $8 \%$ and $23 \%$ chord (Figure 35, Figure 36). The high-frequency content can still be noted in ZDES simulations (Figure 35 and Figure $36 \mathrm{~d}$ and e), showing that the captured turbulent fluctuations also affect the URANS part of the simulation. As before, this part of the spectrum is modeled by DRSM and therefore it is missing in the corresponding spectra (Figure 35 and Figure $36 \mathrm{c}$ ). However, both ZDES and DRSM spectra also show a lower frequency peak for a Strouhal number in the range $S t=8-13$ at $8 \%$ chord, decreasing to $S t=4-5$ at $23 \%$ chord. This last value is very close to the one obtained in the shear layer at the same chordwise location, so that this peak can be attributed to the fluctuation of the recirculation bubble due to the Kelvin-Helmholtz instability. The probe located at $8 \%$ chord also shows a large peak for a Strouhal number of the order of 2, corresponding to the oscillation of loads due to vortex shedding, which can also be guessed in both ZDES spectra, probably because the magnitude of the turbulent spectra is smaller close to the wall where the method operates in URANS mode. Again, scale separation between these low- 
frequency unsteady phenomena and turbulence is valid, as turbulence applies to Strouhal numbers larger than 30 .

a) DRSM

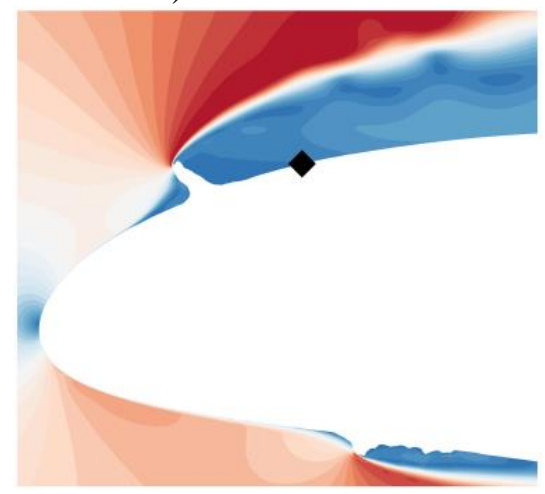

c) RANS spectrum

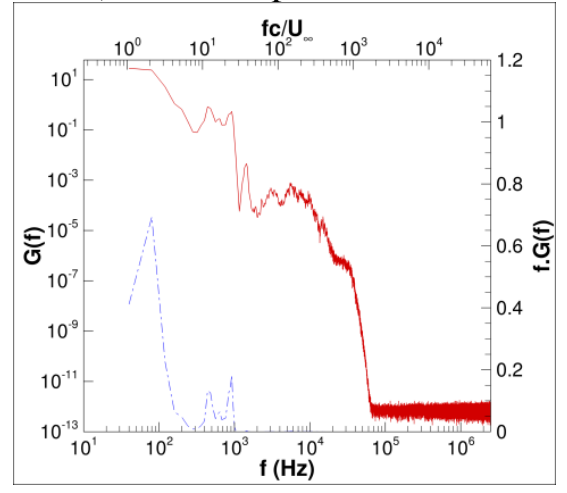

b) ZDES

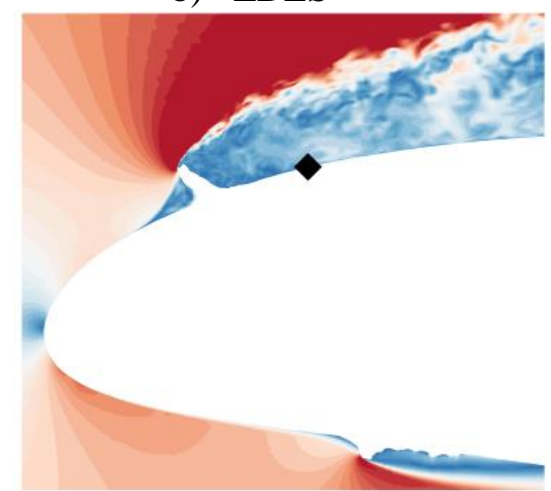

e) Mesh II spectrum

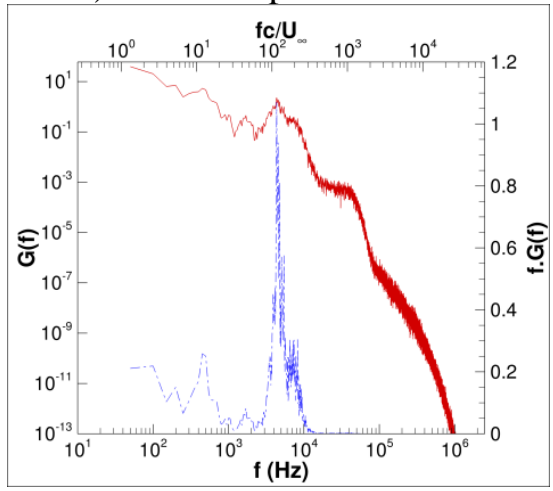

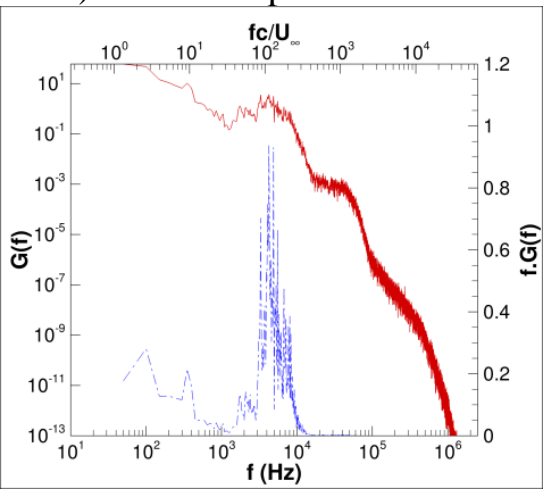

Figure 35: Pressure spectra near the wall at $8 \%$ chord - top row: position of probe with velocity contours; bottom row: spectra for DRSM (left) and ZDES mesh I (middle) and II (right) 
a) DRSM

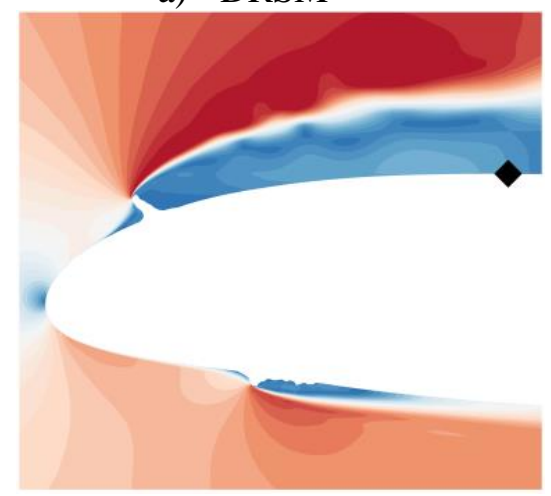

c) RANS spectrum

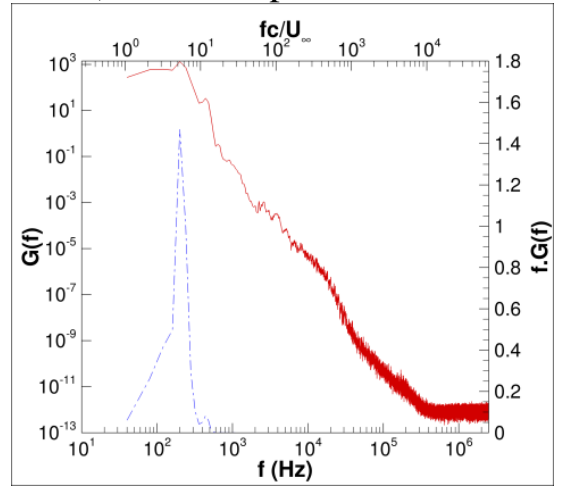

b) ZDES

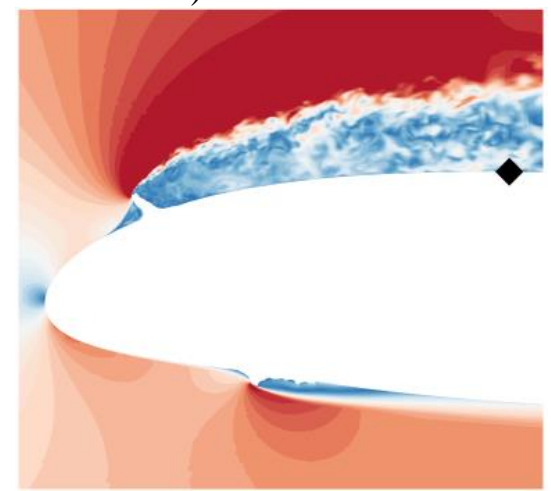

d) Mesh I spectrum

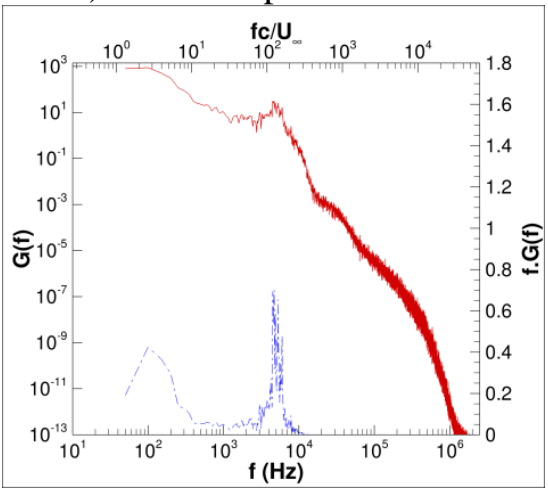

e) Mesh II spectrum

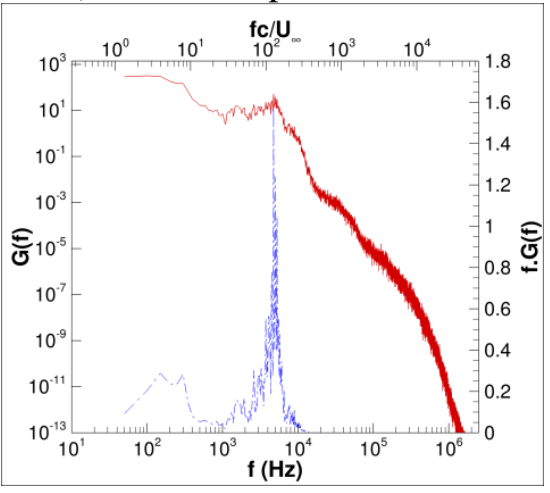

Figure 36: Pressure spectra near the wall at 23\% chord - top row: position of probe with velocity contours; bottom row: spectra for DRSM (left) and ZDES mesh I (middle) and II (right)

\section{Synthesis}

To conclude, beside the filtering effect of RANS resulting in a much richer frequency spectra of the ZDES solutions, the unsteady phenomena captured by DRSM qualitatively fit the low-frequency content of ZDES. However, DRSM results tend to concentrate the unsteady part of the solution at a few discrete frequencies, while ZDES spreads it in a wider domain in the same range of frequencies. This lowfrequency content is mainly induced by the shear layer instability downstream the ridge ice shape, which governs the vortex shedding downstream as well as the fluctuations of the recirculation bubble. Such phenomena interact with the turbulent content of the flow, as a small overlap of the turbulent scales with the larger frequencies computed by DRSM could be observed. However, scale separation between the low-frequency shedding and turbulence seem to work reasonably well. 


\section{Conclusions}

This article presented a numerical investigation of the capability to predict the flow around a NACA 23012 airfoil with two ice shapes, a spanwise ridge and leading edge roughness. Several turbulence models have been tested (Spalart-Allmaras, Menter k- $\omega$ SST, Hellsten EARSM k- $\omega$ and DRSM SSG- $\omega$ and SSG/LRR- $\omega$ ) as well as the ZDES approach. Most of the simulations were performed with a grid conforming to the actual ice shape, thus adding the difficulty of generating good quality structured grids around such complex shapes.

In spite of its deficiencies in predicting some details of the flow, the SA model has proven to be numerically very robust and efficient, converging to steady states for all tested configurations. It also provides a fair description of the complex flow separation phenomena over the full domain of interest of the airfoil. The roughness case also allowed evaluating the roughness model applied to a clean airfoil grid, showing satisfactory results once the equivalent sand grain height has been determined. In the present work, the results with a body conforming grid at one single angle of attack were sufficient for estimating it.

For the spanwise ridge, the SST and EARSM models were also tested. They require a timeconsistent method to converge to steady state, which is far from efficient. Furthermore, they generally provide too early stall with respect to the other models.

The DRSM model was more difficult to apply to such kind of geometries, although significant progress in terms of robustness and applicability was obtained by switching from a SSG- $\omega$ formulation to a SSG/LRR- $\omega$ one. One difficulty associated with this kind of model is that, being less dissipative than traditional Boussinesq models, unsteady solutions are frequently obtained as soon as large flow separation occurs, so that the computations have to be run in a time-accurate mode. The unsteady solution has to be subsequently time-averaged for comparison with experiment. Although it is clear that such kind of model requires more extensive validation including a true grid convergence analysis, DRSM predicts iced airfoil aerodynamics and induced performance degradation similarly to SA, but with different qualities and 
deficiencies. The solutions obtained are more expensive in terms of CPU requirements, but they include more physical content, more particularly flow unsteadiness, with low-frequency results comparable to those of ZDES for the spanwise ridge.

The ZDES method was applied only to one angle of attack of the spanwise ridge because of the higher cost of such hybrid RANS-LES simulations. The results obtained are among the best for $\alpha=2^{\circ}$ incidence, very close to the DRSM predictions. However, these two sets of simulations (ZDES and DRSM) also predict a too low suction in the recirculation bubble downstream the ridge ice shape, too strong vortex shedding and too small mean pressure gradient in the reattachment downstream. While for ZDES this might be due to a too narrow extension of the mesh along the span direction, this is not the case for DRSM which is purely two-dimensional. Indeed, it can be suspected that the discrepancy partially comes from three-dimensional effects in the experiment, as the ridge ice shape was actually three-dimensional in the tests.

A comparison of the unsteady solutions computed with DRSM and ZDES was also completed. Although the relatively short period of time used for averaging resulted in a poor accuracy of the lowfrequency content where the comparison can only be qualitative, reasonable statistical convergence could be achieved for higher frequencies. The two modelisations capture vortex shedding dowstream the recirculation in the same low-frequency range, resulting in similar effects on the mean flow field. A comparison of the pressure spectra at various locations in the field also indicates that, beside the filtering effect of the URANS approach which does not allow it to capture high-frequency turbulent phenomena, the low-frequency response of both solutions presents similarities with varying characteristic frequencies related to the fluctuations of the recirculation bubble and the shedding of vorticity in the wake of the ridge ice shape. A very good comparison of the Reynolds stress tensor captured by ZDES with the one modelled by DRSM is also obtained.

Finally, these simulations of ice effect on airfoil performance suggest that a geometrically $2 \mathrm{D}$ analysis of the problem may not be appropriate because ice shapes are 3D. This may explain some of the discrepancies observed in the present work. 


\section{Acknowledgements}

This work was performed in the frame of CORICE project funded by DGAC, the French Civil Aviation Agency. The experimental data used in the present work was obtained in the frame of the cooperation between NASA, University of Illinois and ONERA on icing. The authors are also grateful to R. Gaveriaux for his support in the generation of the ZDES meshes, and to S. Deck for his support in signal processing.

\section{References}

${ }^{[1]}$ Lynch, F. T., Khodadoust, A. "Effects of ice accretions on aircraft aerodynamics". Progress in Aerospace Sciences, 37(8), 669-767. (2001).

${ }^{[2]}$ Bragg, M. B., Broeren, A. P., Blumenthal, L. A. "Iced-airfoil aerodynamics". Progress in Aerospace Sciences, 41(5), 323-362. (2005).

${ }^{[3]}$ Chi, X., Zhu, B., Shih, T.I-P., Slater, J.W., Addy, H.E., Choo, Y.K., “Computing aerodynamic performance of a 2D iced airfoil: blocking topology and grid generation”, AIAA paper 2002-0381, 40 Aerospace Sciences Meeting \& Exhibit, 2002.

${ }^{[4]}$ Chung, J., Reehorst, A., Choo, Y., Potapczuk, M., Slater, J., "Navier-Stokes analysis of flowfield characteristics of an ice-contaminated aircraft wing". Journal of aircraft, 37(6), 947-959. (2000).

${ }^{[5]}$ Dunn, T. A., Loth, E., Bragg, M. B. "Computational investigation of simulated large-droplet ice shapes on airfoil aerodynamics”. Journal of aircraft, 36(5), 836-843. (1999).

${ }^{[6]}$ Kumar, S., Loth, E. “Aerodynamic simulations of airfoils with upper-surface ice-shapes”. Journal of aircraft, 38(2), 285-295. (2001).

${ }^{[7]}$ Pan, J., Loth, E. "Reynolds-averaged Navier-Stokes simulations of airfoils and wings with ice shapes". Journal of aircraft, 41(4), 879-891. (2004).

${ }^{[8]}$ Kumar, S., Loth, E. "Detached eddy simulations of an iced-airfoil". AIAA, Aerospace Sciences Meeting and Exhibit, 39 ${ }^{\text {th }}$, Reno, NV. (2001). 
${ }^{[9]}$ Pan, J., Loth, E. "Detached eddy simulations for iced airfoils". Journal of aircraft, 42(6), 14521461. (2005).

[10] Chi, X., Zhu, B., Shih, T.I-P., Addy, H.E., Choo, Y.K., "CFD analysis of the aerodynamics of a business-jet airfoil with leading-edge ice accretion", AIAA paper 2004-0560, 42 ${ }^{\text {nd }}$ Aerospace Sciences Meeting \& Exhibit, 2004.

[11] Alam, M. F., Walters, D. K., Thompson, D. S. "Simulations of separated flow around an airfoil with ice shape using hybrid RANS/LES models". 29th AIAA Applied Aerodynamics Conference. (2011).

[12] Alam, M. F., Thompson, D. S., Walters, D. K. "Hybrid Reynolds-averaged NavierStokes/Large-Eddy Simulation models for flow around an iced wing”. Journal of aircraft, (52(1), 244256. (2015).

[13] Duclercq, M., Brunet, V., Moëns, F., "Physical Analysis of the Separated Flow Around an Iced Airfoil Based on ZDES Simulations", AIAA Paper 2012-2798, $4^{\text {th }}$ AIAA Atmospheric \& Space Environment Conference, 25-28 June 2012, New Orleans (Louisiana).

[14] Zhang, Y., Habashi, W. G., Khurram, R. A. "Zonal Detached-Eddy Simulation of Turbulent Unsteady Flow over Iced Airfoils”. Journal of Aircraft, 1-14. (2015).

[15] Xiao, M., Zhang, Y., Chen, H., "Numerical study of an iced airfoil based on Delayed DetachedEddy Simulation with low dissipation scheme", $9^{\text {th }}$ AIAA Atmospheric and Space Environments Conference. (2017).

[16] Ribeiro, A.F.P., Singh, D., König, B., Fares, E. "On the sall characteristics of iced wings", AIAA paper 2017-1426, 55 ${ }^{\text {th }}$ AIAA Aerospace Sciences Meeting, Grapevine, TX, Jan. 2017.

[17] Fares, E., Duda, B., Ribeiro, A.F.P., König, B. "Scale-resolving simulations using a lattice Boltzmann-based approach", CEAS Aeronautical Journal, 2018.

[18] Bragg, M.B., Broeren, A.P., Addy, H.E., Jr., Potapczuk, M.G., Guffond, D., Montreuil, E., “Airfoil ice-accretion aerodynamics simulation", AIAA paper 2007-0085, 45 ${ }^{\text {th }}$ Aerospace Sciences Meeting \& Exhibit, 2002, and NASA/TM-2008-214830. 
[19] Broeren, A. P., Bragg, M. B., Addy, H. E., Jr., Lee, S., Moens, F., and Guffond, D., "Effect of High-Fidelity Ice-Accretion Simulations on Full-Scale Airfoil Performance”, Journal of Aircraft, Vol. 47, No. 1, Jan-Feb 2010, pp. 240-254.

[20] Costes, M., Moens, F., Brunet, V., "Prediction of iced airfoil aerodynamic characteristics", AIAA paper 2016-1547, 54 ${ }^{\text {th }}$ AIAA Aerospace Sciences Meeting, San Diego, CA, Jan. 2016.

[21] Costes, M., Moens, F., “Advanced prediction of iced airfoil aerodynamics”, AIAA paper 20180782, 56 ${ }^{\text {th }}$ AIAA Aerospace Sciences Meeting, Kissimmee, FL, Jan. 2018.

[22] Costes, M., Moens, F., "Prediction of airfoil performance degradation due to ice accretion", $53^{\text {rd }}$ 3AF International Conference on Applied Aeodynamics, Salon-de-Provence (France), March 26-28 2018.

[23] Cambier, L., Heib, S., and Plot, S., "The ONERA elsA CFD software: input from research and feedback from industry", Mechanics\&Industry, Vol. 14, No 3, Jan. 2013, pp. 159-174.

[24] Liou, M.-S., Edwards, J.R., "AUSM schemes and extensions for low Mach and multiphase flows", 30 ${ }^{\text {th }}$ CFD Von Karman Lecture Series, 1999-03, 1999.

[25] Spalart, P. R., and Allmaras, S. R., “A One-Equation Model for Aerodynamic Flows”, AIAA paper 92-0439, 30th AIAA Aerospace Sciences Meeting and Exhibit, Reno, NV, Jan. 6-9, 1992.

[26] Menter, F. R., "Two-equation eddy-viscosity transport turbulence model for engineering applications", AIAA Journal, Vol. 32, No. 8, pp. 1598-1605, 1994.

[27] Hellsten, A., "New two-equation turbulence model for aerodynamics applications", $\mathrm{PhD}$ Dissertation, Dept. of Mechanical Engineering, Helsinki University of Technology, 2004.

[28] Speziale, C.G., Sarkar, S., Gatski, T.B., "Modelling the Pressure-Strain Correlation of Turbulence: An Invariant Dynamical System Approach”, Journal of Fluid Mechanics, Vol. 227, pp. 245272, June 1991.

[29] Launder, B.E., Reece, G.J., Rodi, W., "Preogress in the development of a Reynolds-stress turbulence closure", J. Fluid Mech., vol. 68, part 3, pp. 537-566, 1975.

[30] Deck, S., "Recent improvements in the Zonal Detached Eddy Simulation (ZDES) formulation", Theor. Comput. Fluid Dyn., Vol. 26, No. 6, pp. 523-550, 2012. 
[31] Deck S, Gand F, Brunet V, Ben Khelil S. 2014 "High-fidelity simulations of unsteady civil aircraft aerodynamics: stakes and perspectives. Application of zonal detached eddy simulation." Phil.Trans.R.Soc.A 372: 20130325. http://dx.doi.org/10.1098/rsta.2013.0325

[32] Spalart PR, Jou W-H, Strelets M, Allmaras SR. 1997. "Comments on the feasibility of LES for wings, and on a hybrid RANS/LES approach". In "Advances in DNS/LES”, ed. C Liu, Z Liu, pp. 137147. Columbus, OH: Greyden Press.

[33] Chung, J., Reehorst, A.L., Choo, Y.K., Potapczuk, M.G., "Effect of airfoil ice shape smoothing on the aerodynamic performance", AIAA paper 98-3242, 34th AIAA/ASME/SAE/ASEE Joint Propulsion Conference and Exhibit, 1998.

[34] Aupoix, B., Spalart, P.R., "Extensions of the Spalart-Allmaras turbulence model to account for wall roughness", Int. Journal of Heat and Fluid Flow 24 (2003), pp. 454-462.

[35] Broeren, A.P., Addy, H.E., Jr., Bragg, M.B., Busch, G.T., Guffond, D., Montreuil, E., “Aerodynamic Simulation of Ice Accretion on Airfoils”, NASA/TP 2011-216929, 2011.

[36] Shin., J., "Characteristics of surface roughness associated with leading-edge ice accretion", Journal of Aircraft, Vol. 33, No. 2 (1996), pp. 316-321.

[37] Welch, P. D., "The Use of Fast Fourier Transform for the Estimation of Power Spectra: A Method Based on Time Averaging over Short Modified Periodograms," IEEE Trans. Audio. Electroacoustic, Vol. AU-15, No. 2, 1967, pp. 70-73.

[38] Huerre, P., Rossi, M., " Hydrodynamic instabilities in open flow”, in Hydrodynamics and Nonlinear Instabilities, edited by C. Godrèche and P. Manneville (Cambridge University Press, 1998), pp. 81-214.

[39] Sagaut, P., Deck, S., Terracol, M., “ Multiscale and multiresolution approaches in turbulence. LES, DES and Hybrid RANS/LES methods: applications and guidelines", Imperial College Press, Second Edition, 2013. 